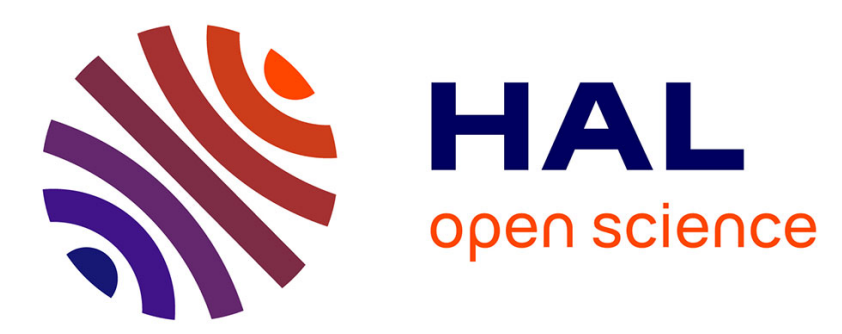

\title{
Block GMRES method with inexact breakdowns and deflated restarting
}

\author{
Emmanuel Agullo, Luc Giraud, Yan-Fei Jing
}

\section{To cite this version:}

Emmanuel Agullo, Luc Giraud, Yan-Fei Jing. Block GMRES method with inexact breakdowns and deflated restarting. [Research Report] RR-8503, INRIA. 2014, pp.31. hal-00963704v2

\section{HAL Id: hal-00963704 \\ https://hal.inria.fr/hal-00963704v2}

Submitted on 26 Mar 2014

HAL is a multi-disciplinary open access archive for the deposit and dissemination of scientific research documents, whether they are published or not. The documents may come from teaching and research institutions in France or abroad, or from public or private research centers.
L'archive ouverte pluridisciplinaire HAL, est destinée au dépôt et à la diffusion de documents scientifiques de niveau recherche, publiés ou non, émanant des établissements d'enseignement et de recherche français ou étrangers, des laboratoires publics ou privés. 
Block GMRES method with inexact breakdowns and deflated restarting

E. Agullo, L. Giraud, Y.-F. Jing

RESEARCH

REPORT

$\mathrm{N}^{\circ} 8503$

March 2014

Project-Teams HiePACS 



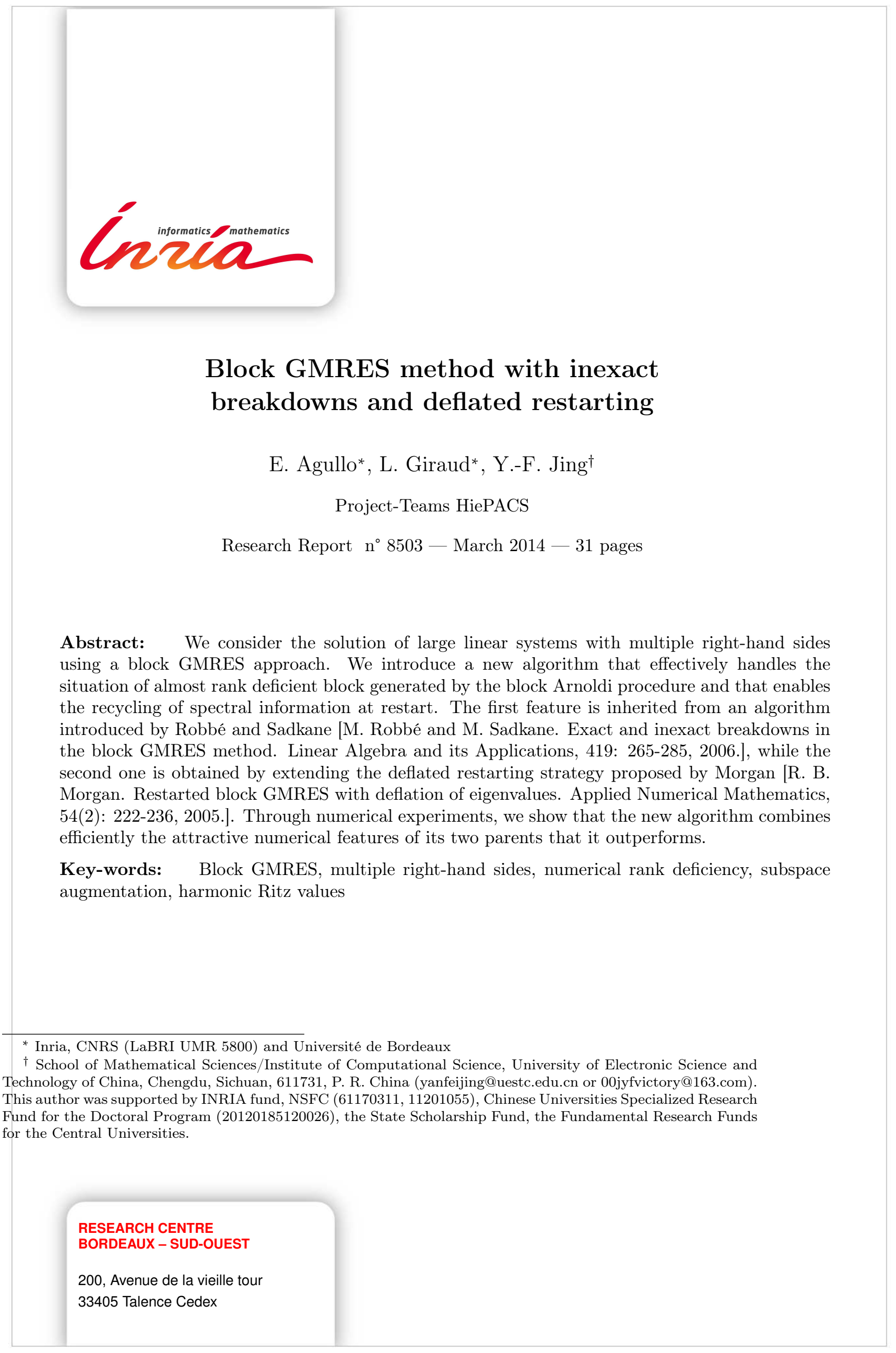




\section{GMRES par bloc avec inexact breakdowns et deflation au restart}

Résumé : Nous considérons la résolution de systèmes linéaires avec second-membres multiplques par une approche GMRES par bloc. Nous introduisons un nouvel algorithme qui gère efficacement d'une part la situation de perte de rang numérique dans les blocs générés par la méthode d'Arnoldi d'autre part le recyclage d'information spectrale au redemarrage via une technique d'augmentation d'espace. La première propriété est hérité de l'algorithme introduit par Robbé and Sadkane [M. Robbé and M. Sadkane. Exact and inexact breakdowns in the block GMRES method. Linear Algebra and its Applications, 419: 265-285, 2006.], et la seconde est obtenue en étendant la stratégie de redémmarage proposée par Morgan [R. B. Morgan. Restarted block GMRES with deflation of eigenvalues. Applied Numerical Mathematics, 54(2): 222-236, 2005.]. Via des expérimentations numériques, nous montrons que ce nouvel algorithme combine efficacement les deux propriétés de ces deux parents dont il améliore les performances.

Mots-clés : bloc GMRES, second-membres multiples, perte de rang numérique, augmentation de sous-espace, valeurs de Ritz harmoniques 


\section{Contents}

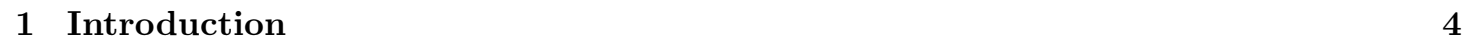

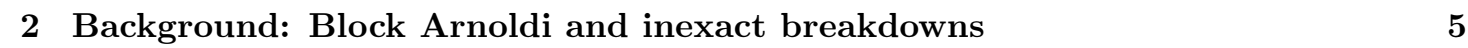

3 Block GMRES with inexact breakdowns and deflated restarting 8

3.1 Analysis of a cycle $\ldots \ldots \ldots \ldots \ldots$

3.1 .1 Harmonic Ritz vectors and residuals $\ldots \ldots \ldots \ldots$. . . . . . . . . . 8

3.1 .2 Block GMRES with inexact breakdowns relations at restart. . . . . . . . . 10

3.1.3 Algorithm for restarting with deflated targeted harmonic Ritz vectors. . . 13

3.1.4 Right-hand sides of the least-squares problem . . . . . . . . . . . . . . 14

3.2 The new block variant $\ldots \ldots \ldots \ldots \ldots \ldots \ldots \ldots$

3.3 Stopping criterion $\ldots \ldots \ldots \ldots \ldots \ldots \ldots \ldots \ldots$

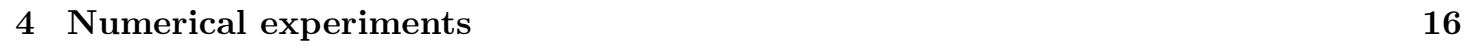

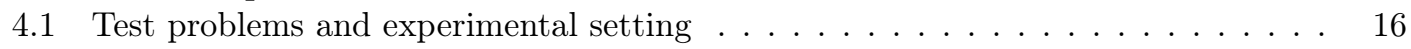

4.2 Some comparisons $\ldots \ldots \ldots \ldots \ldots \ldots \ldots \ldots \ldots$

4.3 Influence of the number of right-hand sides . . . . . . . . . . . . . . . . . . . . . 19

4.4 Inexact breakdowns versus targeted accuracy . . . . . . . . . . . . . . . . . . . . . . . 19

4.5 Further improvement $\ldots \ldots \ldots \ldots \ldots \ldots \ldots \ldots$

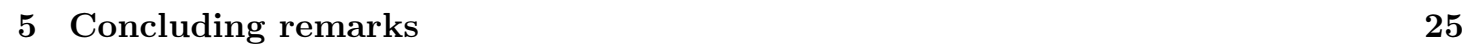

\begin{tabular}{|l|}
\hline A Robbé, Sadkane Block Arnoldi with inexact breakdowns \\
\hline
\end{tabular}

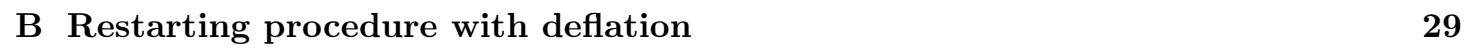

C Alpha variant of BGMRES with inexact breakdowns and deflated restarting 31 


\section{Introduction}

We consider the solution of the linear system with $p$ right-hand sides given simultaneously

$$
A X=B,
$$

where, $A \in \mathbb{C}^{n \times n}$ is a square nonsingular matrix of large dimension $n, B=\left[b^{(1)}, b^{(2)}, \ldots, b^{(p)}\right] \epsilon$ $\mathbb{C}^{n \times p}$ are the given right-hand sides of full rank with $p \ll n$, and $X \in \mathbb{C}^{n \times p}$ are the solutions to be computed. Many large scientific and industrial applications, such as in radar cross section calculation in electromagnetism, wave scattering and wave propagation in acoustics, various source locations in seismic and parametric studies in general, require the solution of a sequence of linear systems with several right-hand sides given simultaneously. In that framework, block Krylov approaches appear as good candidates for the solution as the Krylov subspaces associated with each right-hand side are shared to enlarge the search space. They are attractive not only because of this numerical feature (larger search subspace), but also from a computational view point as they enable the use of BLAS3 like implementation. Their block-structures exhibit nice properties with respect to data locality and re-usability that comply with the memory constraint of modern multicore architectures. For a recent survey on block Krylov subspace methods, we refer to [18.

One difficulty when considering block Krylov methods is the situation when for a presribed accuracy some right-hand sides converge much faster than others or when a linear combination of right-hand sides converges. In order to ensure the robustness of the numerical scheme and reduce the computational effort, a deflation strategy should be implemented to manage the space expansion [13. In a block Arnoldi context, an exact convergence translates into a rank deficiency of the block of vectors to be used to expand the space and consequently leads to a breakdown (which contrary to the non-block counterpart is not a happy breakdown). To address convergence at a targeted numerical threshold, Robbé and Sadkane [33] introduced the idea of the inexact breakdowns. Instead of discarding some directions for the next space expansion, technique that might slow down the convergence [22, they keep the related information of subspaces spanned by these vectors and possibly reintroduce them later. They derive an algorithm that still exhibits a block Arnoldi-like recursion and they propose two criteria ( $W$-criterion and $R$-criterion) to detect the inexact breakdowns to carry out BGMRES. They show that the $R$-criterion is more advisable to use than the $W$-criterion because it can efficiently detect inexact breakdowns when BGMRES is restarted and this criterion can be closely related to the backward error used to define the stopping criterion.. Therefore, only the $R$-criterion is considered in this paper; the corresponding BGMRES using this $R$-criterion to detect Inexact Breakdowns is referred to as IB-BGMRES.

It is well known that convergence of Krylov subspace methods for systems of linear equations depends to a large degree on the distribution of eigenvalues. Different numerical techniques have been proposed in the GMRES context to alleviate the effect of part of the spectrum either through preconditioning updates [15, or via information recycling at restart [14, 19]. In many approaches, some estimate of the invariant subspace is searched in the Krylov subspace and reused in the next restart either by augmenting the space [6, 26, 34], by deflating over the subspace [27] or by ensuring some orthogonality properties with respect to that space [31]. One of the most recent work in this field based on a deflation approach is GMRES-DR [27] extended to block-GMRES (referred to as BGMRES-DR) in 28]. This proposed BGMRES-DR is shown to perform much better than standard block-GMRES (referred to as BGMRES) [36, 42].

In this paper we study and design a block variant that combines effectively the key features of IB-BGMRES and BGMRES-DR. Numerical experiments demonstrate that this approach substantially keeps the efficiency of BGMRES-DR by augmenting the space with directions associ- 
ated with some approximations of selected eigenvalues while it allows a decrease of the computational cost thanks to the detection and proper managment of partial convergences. For other variants of BGMRES, we refer to the rich literature, for instance [1, 2, 15, 7, 8, 17, 20, 23, 24, 32, 34, 38, 37.

The remainder of this paper is organized as follows. In Section 2 , the block Arnoldi process [18, [33, 35, 40] and some insights of IB-BGMRES are firstly reviewed. Some fundamental properties, similar to those of BGMRES-DR, are established in Section 3.1.1 for the harmonic residual vectors as well as their relationships with the residual vectors of the linear systems. In Section 3.1.2 we derive the new restarting procedure that enables to start with a subspace containing the selected spectral information and the linear system residuals. At restart, the new Arnoldi recursion for the new starting subspace is computed at low computational cost without involving any additional matrix-vector product with $A$. We show in Section 3.1.4 how the inexact breakdowns affect the right-hand sides of the least-squares problems and how they can be incrementally computed (contrarily to classical implementations where these right-hand sides are just expanded with zero entries to account for the increase of the Krylov space). The new block variant - IBBGMRES-DR is presented in Section 3.2. In Section 3.3, we discuss the stopping criterion and its consequences on quality of the computed solutions by IB-BGMRES-DR in a backward error framework. Numerical experiments on a set of typical test problems are reported in Section 4 and concluding remarks are discussed in Section 5

The symbol $\|\cdot\|_{q}$ denotes the Euclidean norm when $q=2$ and the Frobenius norm when $q=F$. The superscript " $H$ " denotes the transpose conjugate of a vector or matrix. For convenience of algorithm illustration and presentation, some MATLAB notation is used. A subscript " $k$ " for a scalar or a matrix is used to indicate that the scalar or the matrix is obtained at iteration $k$. A matrix $C \in \mathbb{C}^{k \times \ell}$ consisting of $k$ rows and $\ell$ columns sometimes is denoted as $C_{k \times \ell}$ explicitly. The identity and null matrices of dimension $k$ are denoted respectively by $I_{k}$ and $0_{k}$ or just $I$ and 0 when the order is evident from the context. If $C \in \mathbb{C}^{k \times \ell}$, the singular values of $C$ are denoted by $\sigma_{1}(C) \geq \ldots \geq \sigma_{\min (k, \ell)}(C)$ in descent order.

\section{Background: Block Arnoldi and inexact breakdowns}

Denote by $X_{0}$ the initial block guesses, and by $R_{0}=B-A X_{0}$ the corresponding nonsingular initial block residuals. Block GMRES builds the Krylov space $\mathscr{K}_{j}\left(A, R_{0}\right)=\operatorname{span}\left(R_{0}, A R_{0}, \ldots, A^{j-1} R_{0}\right)$ and searches for approximated solutions that minimize the 2-norms of the residuals associated with the individual right-hand sides. When one solution or a linear combination of the solutions has converged to the target accuracy, the block-Arnoldi procedure implemented to build an orthonormal basis of $\mathscr{K}_{j}\left(A, R_{0}\right)$ needs to be modified to account for this partial convergence. This partial convergence is characterized by a numerical rank deficiency in the new $p$ directions one attempts to introduce for enlarging the Krylov space. In [33], the authors present an elegant numerical variant that enables the detection of what is referred to as inexact breakdowns. In that approach the directions that have a low contribution to the residual block are discarded from the set of vectors used to expand the space at the next iteration. In this section, we try to give an insight and the main equalities required to derive the IB-BGMRES-DR algorithm. We refer the reader to the original paper [33] for a detailed and complete description. For the sake of simplicity of exposure and easy cross-reading, we adopt most of the notations from [33.

Let $R_{0}=V_{1} \Lambda_{1}$ be the reduced $Q R$-factorization of $R_{0}$, where $V_{1} \in \mathbb{C}^{n \times p}$ is matrix with orthonormal columns and $\Lambda_{1} \in \mathbb{C}^{p \times p}$ is an upper triangular full rank matrix. Then an orthonormal basis of block Krylov subspace

$$
\mathscr{K}_{j}\left(A, V_{1}\right)=\operatorname{span}\left(V_{1}, A V_{1}, \ldots, A^{j-1} V_{1}\right),
$$


can be constructed using a block Arnoldi process described in Algorithm 1 .

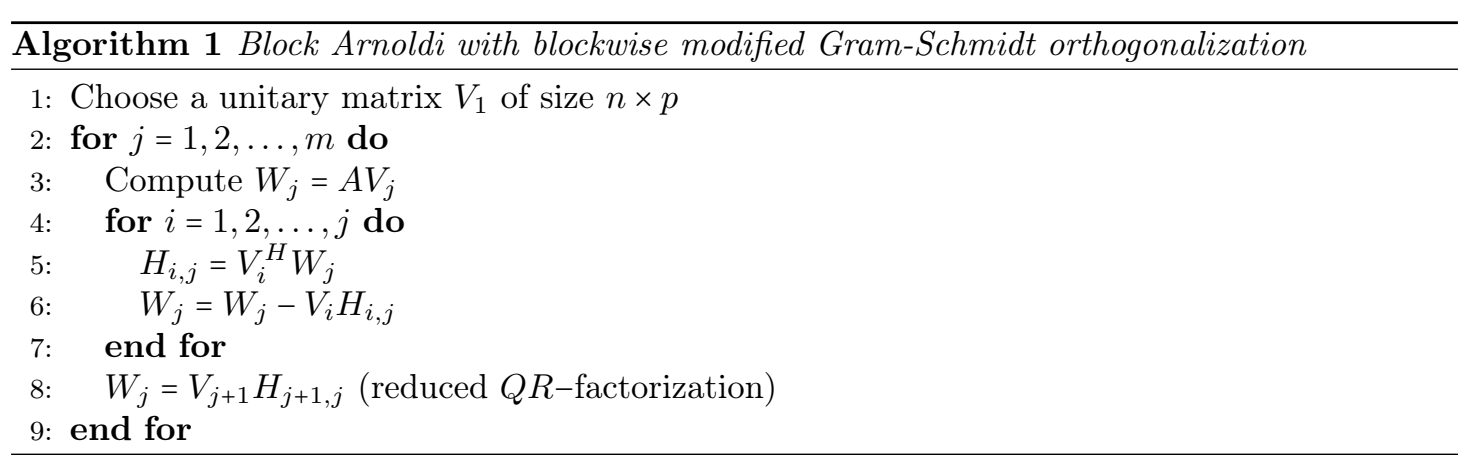

Because when an inexact breakdown occurs, not all the space spanned by $W_{j}$ is considered to build $V_{j+1}$ in order to expand the space (Step 8 of the Algorithm 1), where a subscript $j$ is added to denote its block number of columns. Let $p_{1}=p$ and denote by $p_{j+1}$ the column rank of the block orthonormal basis vector $V_{j+1}$. Then $V_{j+1} \in \mathbb{C}^{n \times p_{j+1}}, W_{j} \in \mathbb{C}^{n \times p_{j}}$ and $H_{j+1, j} \in \mathbb{C}^{p_{j+1} \times p_{j}}$. As a consequence the dimension of the search space $\mathscr{K}_{j}\left(A, V_{1}\right)$ considered at the $j^{t h}$ iteration is no longer necessarily equal to $j \times p$ but is equal to $n_{j}=\sum_{i=1}^{j} p_{i}$; that is, the sum of the column ranks of $V_{i}^{\prime} s(i=1, \ldots, j) . \mathscr{V}_{j}=\left[V_{1}, \ldots, V_{j}\right] \in \mathbb{C}^{n \times n_{j}}\left(\mathscr{V}_{j+1}=\left[\mathscr{V}_{j}, V_{j+1}\right]\right)$ denotes the orthonormal basis of $\mathscr{K}_{j}\left(A, V_{1}\right)$ (respectively $\mathscr{K}_{j+1}\left(A, V_{1}\right)$ ). Associated with these bases we define $\underline{\mathscr{H}}_{j}=$ $\left[\begin{array}{c}\mathscr{H}_{j} \\ 0 \ldots 0 H_{j+1, j}\end{array}\right] \in \mathbb{C}^{n_{j+1} \times n_{j}}$ the block upper Hessenberg matrix, where $\mathscr{H}_{j}=\left(H_{i, \ell}\right)_{1 \leq i, \ell \leq j} \in \mathbb{C}^{n_{j} \times n_{j}}$.

When no inexact breakdown has occured $p_{j+1}=p_{j}=\ldots=p_{1}=p$, the range of $W_{j}$ has always been used to enlarge the search space. Consequently, one obtains the standard block Arnoldi relation 35] from Algorithm 1]

$$
A \mathscr{V}_{j}=\mathscr{V}_{j} \mathscr{H}_{j}+\left[0_{n \times n_{j-1}}, \quad W_{j}\right]=\mathscr{V}_{j+1} \underline{\mathscr{H}}_{j}
$$

The block minimum residual norm approaches, such as BGMRES [36, 42] build approximations to the solutions $X$ at iteration $j$ of the form

$$
X_{j}=X_{0}+\mathscr{V}_{j} Y_{j}
$$

where $Y_{j}$ solves the least-squares problem

$$
\left\|B-A X_{j}\right\|_{F}=\min _{Y \in \mathbb{C}^{n_{j} \times p}}\left\|\mathscr{V}_{j+1}\left(\Lambda_{j}-\underline{\mathscr{H}_{j}} Y\right)\right\|_{F}=\min _{Y \in \mathbb{C}^{n_{j} \times p}}\left\|\Lambda_{j}-\underline{\mathscr{H}_{j}} Y\right\|_{F}
$$

because $\mathscr{V}_{j+1}$ forms an orthonormal basis and $\Lambda_{j}=\left[\begin{array}{c}\Lambda_{1} \\ 0\end{array}\right] \in \mathbb{C}^{n_{j+1} \times p}$.

To account for a numerical deficiency in the residual block $R_{j}=B-A X_{j}$ in a way that is described later, Robbé and Sadkane 33] proposed to split

$$
W_{j}=V_{j+1} H_{j+1, j}+Q_{j} .
$$

so that $Q_{j}$ and $V_{j+1}$ are orthogonal and only $V_{j+1}$ is used to enlarge $\mathscr{V}_{j}$ to form $\mathscr{V}_{j+1}$. We can then extend Equation (2) into

$$
A \mathscr{V}_{j}=\mathscr{V}_{j} \mathscr{H}_{j}+\left[\mathcal{Q}_{j-1}, \quad W_{j}\right],
$$

where $\mathcal{Q}_{j-1}=\left[Q_{1}, \ldots, Q_{j-1}\right] \in \mathbb{C}^{n \times n_{j-1}}$ accounts for all the abandoned directions. The matrix $\mathcal{Q}_{j-1}$ is rank deficient, that reduces to zero matrix of $\mathbb{C}^{n \times n_{j-1}}$ when no inexact breakdown has occured; in that case Equation (4) reduces to Equation (2). 
In order to characterize a minimum norm solution in the space spanned by $\mathscr{V}_{j}$ using Equation (4) we need to form an orthonormal basis of the space spanned by $\left[\mathscr{V}_{j}, \mathcal{Q}_{j-1}, W_{j}\right]$. This is performed by first orthogonalizing $\mathcal{Q}_{j-1}$ against $\mathscr{V}_{j}$, that is $\tilde{\mathcal{Q}}_{j-1}=\left(I-\mathscr{V}_{j} \mathscr{V}_{j}^{H}\right) \mathcal{Q}_{j-1}$. Because $\mathcal{Q}_{j-1}$ is low rank so is $\tilde{\mathcal{Q}}_{j-1}$ that can be written

$$
\tilde{\mathcal{Q}}_{j-1}=P_{j-1} G_{j-1} \text { with }\left\{\begin{array}{l}
P_{j-1} \in \mathbb{C}^{n \times \tilde{q}_{j-1}} \text { has orthonormal columns with } \mathscr{V}_{j}^{H} P_{j-1}=0, \\
G_{j-1} \in \mathbb{C}^{\tilde{q}_{j-1} \times n_{j-1}} \text { is of full rank. }
\end{array}\right.
$$

Next $W_{j}$, that is already orthogonal to $\mathscr{V}_{j}$, is made to be orthogonal to $P_{j-1}$ with $W_{j}-P_{j-1} C_{j}$ where $C_{j}=P_{j-1}^{H} W_{j}$; then one computes $\tilde{W}_{j} D_{j}$ the reduced QR factorization of $W_{j}-P_{j-1} C_{j}$. Eventually, the columns of the matrix $\left[\mathscr{V}_{j}, P_{j-1}, \tilde{W}_{j}\right]$ form an orthonormal basis of the space spanned by $\left[\mathscr{V}_{j}, \mathcal{Q}_{j-1}, W_{j}\right]$.

With this new basis Equation (4) writes

$$
A \mathscr{V}_{j}=\mathscr{V}_{j} \mathscr{L}_{j}+\left[P_{j-1} G_{j-1},\left[P_{j-1}, \tilde{W}_{j}\right]\left[\begin{array}{c}
C_{j} \\
D_{j}
\end{array}\right]\right]=\left[\mathscr{V}_{j}, P_{j-1}, \tilde{W}_{j}\right]\left[\begin{array}{cc}
\mathscr{L}_{j} \\
G_{j-1} & C_{j} \\
0 & D_{j}
\end{array}\right]
$$

where $\mathscr{L}_{j}=\left[\begin{array}{lllll}H_{1,1} & \cdots & \cdots & \cdots & H_{1, j} \\ H_{2,1} & \ddots & & & \vdots \\ V_{3}^{H} Q_{1} & \ddots & \ddots & & \vdots \\ \vdots & \ddots & \ddots & \ddots & \vdots \\ V_{j}^{H} Q_{1} & \cdots & V_{j}^{H} Q_{j-2} & H_{j, j-1} & H_{j, j}\end{array}\right] \in \mathbb{C}^{n_{j} \times n_{j}}$ is no longer upper Hessenberg as soon as one inexact breakdown occurs, i.e., $\exists \ell Q_{\ell} \neq 0$.

The least-squares problem to be solved to compute the minimum norm solution associated with the generalized Arnoldi Equation (6) becomes

$$
Y_{j}=\underset{Y \in \mathbb{C}^{n_{j} \times p}}{\operatorname{argmin}}\left\|\Lambda_{j}-\mathscr{F}_{j} Y\right\|_{F},
$$

with

$$
\mathscr{F}_{j}=\left[\begin{array}{cc}
\mathscr{L}_{j} \\
G_{j-1} & C_{j} \\
0 & D_{j}
\end{array}\right] \quad \text { and } \quad \Lambda_{j}=\left[\begin{array}{c}
\Lambda_{1} \\
0 \\
0
\end{array}\right]
$$

so that Equation (4) writes in a more compact form as

$$
A \mathscr{V}_{j}=\left[\mathscr{V}_{j},\left[P_{j-1}, \tilde{W}_{j}\right]\right] \mathscr{F}_{j} .
$$

Robbé and Sadkane [33, introduced the so-called R-criterion to extract from the $\left[P_{j-1}, \tilde{W}_{j}\right]$ the subspace to be used to build $V_{j+1}$ as follows. Based on the SVD of the projected residual $\Lambda_{j}-\mathscr{F}_{j} Y_{j}=\mathbb{U}_{1} \Sigma_{1} \mathbb{V}_{1}^{H}+\mathbb{U}_{2} \Sigma_{2} \mathbb{V}_{2}^{H}$ where $\Sigma_{1}$ contains the singular values larger than a prescribed threshold $\epsilon^{(R)}$, they decompose $\mathbb{U}_{1}=\left(\begin{array}{l}\mathbb{U}_{1}^{(1)} \\ \mathbb{U}_{1}^{(2)}\end{array}\right)$ in accordance with $\left[\mathscr{V}_{j},\left[P_{j-1}, \tilde{W}_{j}\right]\right]$, and consider $\left[\mathbb{W}_{1}, \mathbb{W}_{2}\right]$ unitary so that Range $\left(\mathbb{W}_{1}\right)=\operatorname{Range}\left(\mathbb{U}_{1}^{(2)}\right)$. The new set of vectors selected to expand the search space

$$
V_{j+1}=\left[P_{j-1}, \tilde{W}_{j}\right] \mathbb{W}_{1}
$$

is the one that contributes the most to the residual. Through this mechanism, directions that have been abandoned at a given iteration can be reintroduced, if the residual block has a large component along them. Furthermore, this selection strategy ensures that all the solutions have 
converged when $p$ inexact breakdowns have been detected; we refer to Section 3.3 for the discussion on how $\epsilon^{(R)}$ should be defined to ensure a convergence of all the solutions to a prescribed backward error.

We do not give the details of the calculation and refer to [33] for a complete description, but only state that via this decomposition the main terms that appear in Equation (6) can be computed incrementally from one iteration to the next as follows:

- based on the following recurrence $\mathscr{L}_{j}=\left[\begin{array}{cc}H_{1, j} \\ \mathscr{L}_{j-1} \\ \vdots \\ H_{j, j}\end{array}\right]$ where $\underline{L}_{j-1}=\left[\begin{array}{c}\mathscr{L}_{j-1} \\ V_{j}^{H} \mathcal{Q}_{j-2} H_{j, j-1}\end{array}\right]$ the last block row of $\underline{L}_{j}$ at next iteration $(j+1)$ is given by $\mathscr{L}_{j+1,:}=\mathbb{W}_{1}^{H}\left[\begin{array}{cc}G_{j-1} & C_{j} \\ 0 & D_{j}\end{array}\right]$. The last block column of $\mathscr{L}_{j+1}$ results from the block Arnoldi orthogonalization. Those matrices play a central role in IB-BGMRES since they define the procedure to enlarge the space and follow the equation below:

$$
A \mathscr{V}_{j}=\mathscr{V}_{j+1} \underline{\mathscr{L}}_{j}+\tilde{\mathcal{Q}}_{j}
$$

- the new compressed form of the abandoned directions $\tilde{\mathcal{Q}}_{j}$ is given by the new orthonormal set of vectors

$$
P_{j}=\left[P_{j-1}, \tilde{W}_{j}\right] \mathbb{W}_{2},
$$

the complementary part of $V_{j+1}$ and their components in the space spanned by $P_{j}$ are $G_{j}=\mathbb{W}_{2}^{H}\left[\begin{array}{cc}G_{j-1} & C_{j} \\ 0 & D_{j}\end{array}\right]$.

\section{Block GMRES with inexact breakdowns and deflated restart- ing}

In this section we present the new block algorithm that effectively handles the inexact breakdowns and that enables the recycling of spectral information at restart. These features are obtained by extending to IB-BGMRES the augmentation strategy of BGMRES-DR.

\subsection{Analysis of a cycle}

We discuss now the two main points related to the extension of BGMRES-DR in an inexact breakdown setting: what is the harmonic Ritz information recovered at restart and is it still possible to restart at low computational cost a block Arnoldi-like recurrence formula similar to Equation (7)? Both issues will be addressed in the next section.

\subsubsection{Harmonic Ritz vectors and residuals}

In order to describe how a restarting strategy enabling to recycle spectral information can be implemented in combination with the block GMRES with inexact breakdown, we first need to establish some key properties. In particular, we first characterize the subspace to be considered in order to define the harmonic Ritz vectors [16, 25, 29, 30, 39] that will be recycled at restart. These properties enable us to ensure that both the residuals of the linear systems and the harmonic Ritz vectors to be recycled can be represented in a same subspace at restart, while maintaining a block Arnoldi-like relation at low computational cost. 
Definition 1. Harmonic Ritz pair. Consider a subspace $\mathcal{U}$ of $\mathbb{C}^{n}$. Given a matrix $B \in \mathbb{C}^{n \times n}$, $\lambda \in \mathbb{C}$ and $y \in \mathcal{U},(\lambda, y)$ is a harmonic Ritz pair of $B$ with respect to $\mathcal{U}$ if and only if

$$
B y-\lambda y \perp B \mathcal{U}
$$

or equivalently, for the canonical scalar product,

$$
\forall w \in \operatorname{Range}(B \mathcal{U}) \quad w^{H}(B y-\lambda y)=0 .
$$

The vector $y$ is a harmonic Ritz vector associated with the harmonic Ritz value $\lambda$.

Lemma 1 presents the harmonic Ritz formulation used in the block GMRES variant with inexact breakdowns and deflated restarting.

Lemma 1. Let $\mathcal{U}=\operatorname{span}\left(\mathscr{V}_{m}\right)$, where $\mathscr{V}_{m}$ is the orthonormal basis built by IB-BGMRES at the end of a cycle. The harmonic Ritz pairs $\left(\tilde{\theta}_{i}, \tilde{g}_{i}\right)$ associated with $\mathscr{V}_{m}$ satisfy the following property

$$
\mathscr{F}_{m}^{H}\left(\mathscr{F}_{m} \tilde{g}_{i}-\tilde{\theta}_{i}\left[\begin{array}{c}
\tilde{g}_{i} \\
0_{p}
\end{array}\right]\right)=0,\left(i=1, \ldots, n_{m}\right),
$$

where $\mathscr{F}_{m}$ is defined by Equation (7), $\tilde{g}_{i} \in \mathbb{C}^{n_{m}}$, and $\mathscr{V}_{m} \tilde{g}_{i}\left(i=1, \ldots, n_{m}\right)$ are the harmonic Ritz vectors associated with the corresponding harmonic Ritz values $\tilde{\theta}_{i}\left(i=1, \ldots, n_{m}\right)$.

Proof. Assume that we have performed a first cycle of block Arnoldi with inexact breakdowns (see Algorithm 3). By Petrov-Galerkin condition defined in Definition 1, the harmonic Ritz pairs satisfy

$$
\left(A \mathscr{V}_{m}\right)^{H}\left(A \mathscr{V}_{m} \tilde{g}_{i}-\tilde{\theta}_{i} \mathscr{V}_{m} \tilde{g}_{i}\right)=0,\left(i=1, \ldots, n_{m}\right) .
$$

Substituting (7) with $j=m$ into $(12)$, we get

$$
\mathscr{F}_{m}^{H}\left[\mathscr{V}_{m},\left[P_{m-1}, \tilde{W}_{m}\right]\right]^{H}\left(\left[\mathscr{V}_{m},\left[P_{m-1}, \tilde{W}_{m}\right]\right] \mathscr{F}_{m} \tilde{g}_{i}-\tilde{\theta}_{i} \mathscr{V}_{m} \tilde{g}_{i}\right)=0, \quad\left(i=1, \ldots, n_{m}\right),
$$

which gives the desired result because $\left[\mathscr{V}_{m},\left[P_{m-1}, \tilde{W}_{m}\right]\right]$ has orthonormal columns.

$$
\text { Because } \mathscr{F}_{m}=\left[\begin{array}{c}
\mathscr{L}_{\mathrm{m}} \\
\mathbb{H}_{m}
\end{array}\right] \text { with } \mathbb{H}_{m}=\left[\begin{array}{cc}
G_{m-1} & C_{m} \\
0 & D_{m}
\end{array}\right] \in \mathbb{C}^{p \times n_{m}} \text {, it also reads }
$$

$$
\left(\mathscr{L}_{m}^{H} \mathscr{L}_{m}+\mathbb{H}_{m}^{H} \mathbb{H}_{m}\right) \tilde{g}_{i}=\tilde{\theta}_{i} \mathscr{L}_{m}^{H} \tilde{g}_{i}, i=1, \ldots, n_{m}
$$

Therefore, we compute the $k$ targeted eigenpairs $\left(\tilde{\theta}_{i}, \tilde{g}_{i}\right)$ of the matrix $\mathscr{L}_{m}+\mathscr{L}_{m}^{-H} \mathbb{H}_{m}^{H} \mathbb{H}_{m}$ (see Step 3 of Algorithm 2). In practice, it might be more stable to solve the generalized eigenvalue problem in Equation (13) if $\mathscr{L}_{m}$ is nearly singular.

In Lemma 2, we detail a useful relation as a basis for Proposition 1 showing that the residuals of the linear systems and the residuals of the harmonic Ritz vectors to be recycled can be represented in the same subspace at restart.

Lemma 2. Assume that $\mathscr{L}_{m}$ is of full rank after performing a first cycle of Algorithm 3, then the column vectors $\left(\mathscr{F}_{m} \tilde{g}_{i}-\tilde{\theta}_{i}\left[\begin{array}{c}\tilde{g}_{i} \\ 0\end{array}\right]\right) \in \mathbb{C}^{n_{m}+p}\left(i=1, \ldots, n_{m}\right)$ are all contained in the subspace spanned by the least-squares residuals $R_{L S_{m}}=\left(\Lambda_{m}-\mathscr{F}_{m} Y_{m}\right) \in \mathbb{C}^{\left(n_{m}+p\right) \times p}$, i.e., $\exists \alpha_{i} \in \mathbb{C}^{p}$ so that

$$
\mathscr{F}_{m} \tilde{g}_{i}-\tilde{\theta}_{i}\left[\begin{array}{c}
\tilde{g}_{i} \\
0
\end{array}\right]=R_{L S_{m}} \alpha_{i}
$$

$\mathrm{RR} \mathrm{n}^{\circ} 8503$ 
Proof. From the preceding lemma, we have that $\left(\mathscr{F}_{m} \tilde{g}_{i}-\tilde{\theta}_{i}\left[\begin{array}{c}\tilde{g}_{i} \\ 0\end{array}\right]\right) \in \operatorname{Null}\left(\mathscr{F}_{m}^{H}\right),\left(i=1, \ldots, n_{m}\right)$, Furthermore, we have $n_{m}+p=\operatorname{dim}\left(\operatorname{Range}\left(\mathscr{F}_{m}^{H}\right)\right)+\operatorname{dim}\left(\operatorname{Null}\left(\mathscr{F}_{m}^{H}\right)\right)$. Because $\mathscr{L}_{m}$ is assumed of full rank, we have $\operatorname{dim}\left(\operatorname{Null}\left(\mathscr{F}_{m}^{H}\right)\right)=p$.

On the other hand, $Y_{m}=\operatorname{argmin}_{Y \in \mathbb{C}^{n} m \times p}\left\|\Lambda_{m}-\mathscr{F}_{m} Y\right\|_{F}$, then $\mathscr{F}_{m} Y_{m}$ are the orthogonal projections of $\Lambda_{m}$ on Range $\left(\mathscr{F}_{m}\right)$ and $\left(\Lambda_{m}-\mathscr{F}_{m} Y_{m}\right) \perp \operatorname{Range}\left(\mathscr{F}_{m}\right)$. That is $\left(\Lambda_{m}-\mathscr{F}_{m} Y_{m}\right) \epsilon$ $\operatorname{Null}\left(\mathscr{F}_{m}^{H}\right)$ thanks to Range $\left(\mathscr{F}_{m}\right)^{\perp}=\operatorname{Null}\left(\mathscr{F}_{m}^{H}\right)$, which together with $\operatorname{rank}\left(\Lambda_{m}-\mathscr{F}_{m} Y_{m}\right)=p$ indicates that $\operatorname{span}\left(\Lambda_{m}-\mathscr{F}_{m} Y_{m}\right)=\operatorname{Null}\left(\mathscr{F}_{m}^{H}\right)$. Then consequently $\left(\mathscr{F}_{m} \tilde{g}_{i}-\tilde{\theta}_{i}\left[\begin{array}{c}\tilde{g}_{i} \\ 0\end{array}\right]\right) \in \operatorname{span}\left(\Lambda_{m}-\right.$ $\left.\mathscr{F}_{m} Y_{m}\right),\left(i=1, \ldots, n_{m}\right)$, yielding Equation (14).

Using Lemma 2 and Equation (7) for $j=m$, we can characterize the relationship between harmonic residuals and linear systems residuals in the inexact breakdown framework. This result can be viewed as a generalization of [28, Lemma 2] when inexact breakdowns occur and are handled.

Proposition 1. The harmonic residual vectors are all linear combinations of the residual vectors from the minimum residual solutions of the linear equation problem after performing a first cycle of Algorithm 3 .

Proof. After performing a first cycle of Algorithm 3, we have obtained the orthonormal ma$\operatorname{trix}\left[\mathscr{V}_{m},\left[P_{m-1}, \tilde{W}_{m}\right]\right]$ and the relation $A \mathscr{V}_{m}=\left[\mathscr{V}_{m},\left[P_{m-1}, \tilde{W}_{m}\right]\right] \mathscr{F}_{m}$ from Equation 77 . The harmonic residual vectors $R_{\mathrm{har}}^{(i)}=A \mathscr{V}_{m} \tilde{g}_{i}-\tilde{\theta}_{i} \mathscr{V}_{m} \tilde{g}_{i}\left(i=1, \ldots, n_{m}\right)$ and the residual vectors $R_{m}=B-A X_{m}$ from the minimum residual solutions of the linear equation problem respectively satisfy

$$
\begin{aligned}
& R_{\text {har }}^{(i)}=A \mathscr{V}_{m} \tilde{g}_{i}-\tilde{\theta}_{i} \mathscr{V}_{m} \tilde{g}_{i}=\left[\mathscr{V}_{m},\left[P_{m-1}, \tilde{W}_{m}\right]\right]\left(\mathscr{F}_{m} \tilde{g}_{i}-\tilde{\theta}_{i}\left[\begin{array}{c}
\tilde{g}_{i} \\
0
\end{array}\right]\right),\left(i=1, \ldots, n_{m}\right), \\
& R_{m}=B-A X_{m}=R_{0}-A \mathscr{V}_{m} Y_{m}=V_{1} \Lambda_{1}-A \mathscr{V}_{m} Y_{m}=\left[\mathscr{V}_{m},\left[P_{m-1}, \tilde{W}_{m}\right]\right] R_{L S_{m}} .
\end{aligned}
$$

Because $\left[\mathscr{V}_{m},\left[P_{m-1}, \tilde{W}_{m}\right]\right]$ is an orthonormal basis, Lemma 2 shows that $R_{\text {har }}$ and $R_{m}$ are in the same p-dimensional space which concludes the proof.

\subsubsection{Block GMRES with inexact breakdowns relations at restart}

Next, we will show that the block Arnoldi-like recurrence formulae (7) and (9) still hold after building initial block orthonormal basis vectors of the new search subspace at each restart combined with refined information associated with the prescribed number $k$ of targeted harmonic Ritz vectors calculated at the end of the previous cycle at low computational cost.

Let us further denote by $\mathscr{V}_{m} \tilde{G}$ the $k$ targeted harmonic Ritz vectors, where $\tilde{G}=\left[\tilde{g}_{1}, \ldots, \tilde{g}_{k}\right] \epsilon$ $\mathbb{C}^{n_{m} \times k}$. First add zero rows of size $p$ to $\tilde{G}$ and then append $R_{L S_{m}}$ to form a new matrix denoted

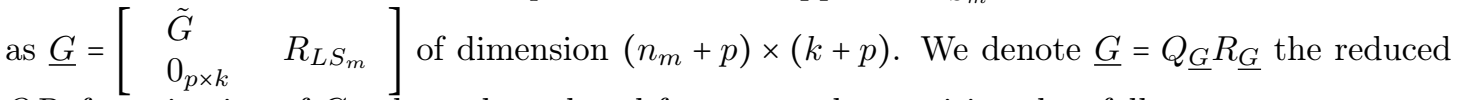
$Q R$-factorization of $\underline{G}$, where the reduced factors can be partitioned as follows

$$
\begin{aligned}
Q_{\underline{G}} & =\left[\begin{array}{ll}
\Gamma_{1} & \Gamma_{2} \\
0_{p \times k} &
\end{array}\right] \in \mathbb{C}^{\left(n_{m}+p\right) \times(k+p)}, \\
R_{\underline{G}} & =\left[\begin{array}{ll}
\Theta_{1} & \\
0_{p \times k} & \Theta_{2}
\end{array}\right] \in \mathbb{C}^{(k+p) \times(k+p)},
\end{aligned}
$$


with $\Gamma_{1}=Q_{\underline{G}}\left(1: n_{m}, 1: k\right), \quad \Gamma_{2}=Q_{\underline{G}}(:, k+1: k+p), \quad \Theta_{1}=R_{\underline{G}}(1: k, 1: k)$ and $\Theta_{2}=R_{\underline{G}}(:, k+1: \bar{k}+p)$ and

$$
\begin{aligned}
& \tilde{G}=\Gamma_{1} \Theta_{1}, \\
& R_{L S_{m}}=Q_{\underline{G}} \Theta_{2} .
\end{aligned}
$$

Proposition 2 shows that the block Arnoldi-like recurrence formulae of IB-BGMRES [33] can be recovered without involving any matrix-vector product with $A$ when restarting with some harmonic information.

Proposition 2. At each restart of block GMRES with inexact breakdowns and deflated restarting, the initial block Arnoldi-like recurrence formulae (7) and (9) still hold in exact arithmetic as

$$
\begin{aligned}
& A \mathscr{V}_{1}^{\text {new }}=\left[\mathscr{V}_{1}^{\text {new }},\left[P_{0}, \tilde{W}_{1}\right]^{\text {new }}\right] \mathscr{F}_{1}^{\text {new }} \\
& A \mathscr{V}_{1}^{\text {new }}=\mathscr{V}_{2}^{\text {new }} \underline{\mathscr{L}}_{1}^{\text {new }}+\tilde{\mathcal{Q}}_{1}^{\text {new }}
\end{aligned}
$$

with

$$
\begin{aligned}
& {\left[\mathscr{V}_{1}^{\text {new }},\left[P_{0}, \tilde{W}_{1}\right]^{\text {new }}\right]=\left[\mathscr{V}_{m},\left[P_{m-1}, \tilde{W}_{m}\right]\right] Q_{\underline{G}},} \\
& R_{0}=\left[\mathscr{V}_{1}^{\text {new }},\left[P_{0}, \tilde{W}_{1}\right]^{\text {new }}\right] \Lambda_{1}^{\text {new }} \text { with } \Lambda_{1}^{\text {new }}=\Theta_{2}, \\
& \mathscr{L}_{1}^{\text {new }}=\Gamma_{1}^{H} \mathscr{L}_{m} \Gamma_{1}, \mathbb{H}_{1}^{\text {new }}=\Gamma_{2}^{H} \mathscr{F}_{m} \Gamma_{1}, \quad \mathscr{F}_{1}^{\text {new }}=\left[\begin{array}{c}
\mathscr{L}_{1}^{\text {new }} \\
\mathbb{H}_{1}^{\text {new }}
\end{array}\right], \\
& V_{2}^{\text {new }}=\left[P_{0}, \tilde{W}_{1}\right]^{\text {new }} \mathbb{W}_{1}^{\text {new }}, P_{1}^{\text {new }}=\left[P_{0}, \tilde{W}_{1}\right]^{\text {new }} \mathbb{W}_{2}^{\text {new }}, \mathscr{L}_{2,:}^{\text {new }}=\mathbb{W}_{1}^{\text {new }} \mathbb{H}_{1}^{\text {new }}, G_{1}^{\text {new }}=\mathbb{W}_{2}^{\text {new }} \mathbb{H}_{1}^{\text {new }}, \\
& \mathscr{V}_{2}^{\text {new }}=\left[\mathscr{V}_{1}^{\text {new }}, V_{2}^{\text {new }}\right], \mathscr{L}_{1}^{\text {new }}=\left[\begin{array}{c}
\mathscr{L}_{1}^{\text {new }} \\
\mathscr{L}_{2,:}^{\text {new }}
\end{array}\right], \tilde{\mathcal{Q}}_{1}^{\text {new }}=P_{1}^{\text {new }} G_{1}^{\text {new }},
\end{aligned}
$$

where $\mathbb{W}_{1}^{\text {new }}$ and $\mathbb{W}_{2}^{\text {new }}$ satisfy

$$
\operatorname{Range}\left(\mathbb{W}_{1}^{\text {new }}\right)=\operatorname{Range}\left(\mathbb{U}_{1}^{\text {new }(2)}\right) \text { with } \mathbb{U}_{1}^{\text {new }}=\left[\begin{array}{c}
\mathbb{U}_{1}^{\text {new }(1)} \\
\mathbb{U}_{1}^{\text {new }}(2)
\end{array}\right] \text { and }\left[\mathbb{W}_{1}^{\text {new }}, \quad \mathbb{W}_{2}^{\text {new }}\right] \text { is unitary }
$$

with

$\Lambda_{1}^{\text {new }}-\mathscr{F}_{1}^{\text {new }} Y_{1}^{\text {new }}=\mathbb{U}_{1}^{\text {new }} \Sigma_{1}^{\text {new }} \mathbb{V}_{1}^{\text {new } H}+\mathbb{U}_{2}^{\text {new }} \Sigma_{2}^{\text {new }} \mathbb{V}_{2}^{\text {new } H}$, where $\sigma_{\min }\left(\Sigma_{1}^{\text {new }}\right) \geq \epsilon^{(R)}>\sigma_{\max }\left(\Sigma_{2}^{\text {new }}\right)$

the SVD to detect inexact breakdown in the restarting block residual where

$$
Y_{1}^{\text {new }}=\underset{Y \in \mathbb{C}^{n_{1} \times p}}{\operatorname{argmin}}\left\|\Lambda_{1}^{\text {new }}-\mathscr{F}_{1}^{\text {new }} Y\right\|_{F} .
$$

Proof. - Show that Equation 19 holds:

From Equation (14) of Lemma 2, we have, $\forall i \in 1, \ldots, k, \exists \alpha_{i} \in \mathbb{C}^{p}$,

$$
\mathscr{F}_{m} \tilde{g}_{i}-\tilde{\theta}_{i}\left[\begin{array}{c}
\tilde{g}_{i} \\
0
\end{array}\right]=R_{L S_{m}} \alpha_{i}
$$

Multiplying both sides of Equation 21 with $\left[\mathscr{V}_{m},\left[P_{m-1}, \tilde{W}_{m}\right]\right]$ gives

$$
\left[\mathscr{V}_{m},\left[P_{m-1}, \tilde{W}_{m}\right]\right] \mathscr{F}_{m} \tilde{g}_{i}-\left[\mathscr{V}_{m},\left[P_{m-1}, \tilde{W}_{m}\right]\right] \tilde{\theta}_{i}\left[\begin{array}{c}
\tilde{g}_{i} \\
0
\end{array}\right]=\left[\mathscr{V}_{m},\left[P_{m-1}, \tilde{W}_{m}\right]\right] R_{L S_{m}} \alpha_{i}
$$


Using (7), we can write the above equation as

$$
A \mathscr{V}_{m} \tilde{g}_{i}=\left[\mathscr{V}_{m},\left[P_{m-1}, \tilde{W}_{m}\right]\right]\left(\tilde{\theta}_{i}\left[\begin{array}{c}
\tilde{g}_{i} \\
0
\end{array}\right]+R_{L S_{m}} \alpha_{i}\right)
$$

which, according to the formulations of $\tilde{G}$ and $\underline{G}$, can be reformulated in a matrix form as

$$
A \mathscr{V}_{m} \tilde{G}=\left[\mathscr{V}_{m},\left[P_{m-1}, \tilde{W}_{m}\right]\right] \underline{G}\left(\begin{array}{c}
\operatorname{diag}\left(\tilde{\theta}_{1}, \ldots, \tilde{\theta}_{k}\right) \\
\alpha_{1}, \ldots, \alpha_{k}
\end{array}\right) .
$$

Using the reduced $Q R$-factorization of $\underline{G}$ and the structure of $\tilde{G}$ as shown in Equation (17), we have

$$
A \mathscr{V}_{m} \Gamma_{1} \Theta_{1}=\left[\mathscr{V}_{m},\left[P_{m-1}, \tilde{W}_{m}\right]\right] Q_{\underline{G}} R_{\underline{G}}\left(\begin{array}{c}
\operatorname{diag}\left(\tilde{\theta}_{1}, \ldots, \tilde{\theta}_{k}\right) \\
\alpha_{1}, \ldots, \alpha_{k}
\end{array}\right) .
$$

Multiplying by $\Theta^{-1}$ from the right-hand side gives

$$
A \mathscr{V}_{m} \Gamma_{1}=\left[\mathscr{V}_{m},\left[P_{m-1}, \tilde{W}_{m}\right]\right] Q_{\underline{G}} R_{\underline{G}}\left(\begin{array}{c}
\operatorname{diag}\left(\tilde{\theta}_{1}, \ldots, \tilde{\theta}_{k}\right) \\
\alpha_{1}, \ldots, \alpha_{k}
\end{array}\right) \Theta_{1}^{-1},
$$

which, from the partition of $Q_{\underline{G}}$ in Equation $\sqrt{15}$, is

$$
A \mathscr{V}_{m} \Gamma_{1}=\left[\mathscr{V}_{m} \Gamma_{1},\left[\mathscr{V}_{m},\left[P_{m-1}, \tilde{W}_{m}\right]\right] \Gamma_{2}\right] R_{\underline{G}}\left(\begin{array}{c}
\operatorname{diag}\left(\tilde{\theta}_{1}, \ldots, \tilde{\theta}_{k}\right) \\
\alpha_{1}, \ldots, \alpha_{k}
\end{array}\right) \Theta^{-1}
$$

On the other hand, Equation 21 can be rewritten in a matrix reformulation as

$$
\mathscr{F}_{m} \tilde{G}-\left(\begin{array}{c}
\tilde{G} \\
0
\end{array}\right) \operatorname{diag}\left(\tilde{\theta}_{1}, \ldots, \tilde{\theta}_{k}\right)=R_{L S_{m}}\left[\alpha_{1}, \ldots, \alpha_{k}\right],
$$

which proceeds similarly to the above manipulations as

$$
\begin{gathered}
\mathscr{F}_{m} \tilde{G}=\underline{G}\left(\begin{array}{c}
\operatorname{diag}\left(\tilde{\theta}_{1}, \ldots, \tilde{\theta}_{k}\right) \\
\alpha_{1}, \ldots, \alpha_{k}
\end{array}\right), \\
\Rightarrow \mathscr{F}_{m} \Gamma_{1} \Theta_{1}=Q_{\underline{G}} R_{\underline{G}}\left(\begin{array}{c}
\operatorname{diag}\left(\tilde{\theta}_{1}, \ldots, \tilde{\theta}_{k}\right) \\
\alpha_{1}, \ldots, \alpha_{k}
\end{array}\right), \\
\Rightarrow Q_{\underline{G}}^{H} \mathscr{F}_{m} \Gamma_{1}=R_{\underline{G}}\left(\begin{array}{c}
\operatorname{diag}\left(\tilde{\theta}_{1}, \ldots, \tilde{\theta}_{k}\right) \\
\alpha_{1}, \ldots, \alpha_{k}
\end{array}\right) \Theta^{-1} .
\end{gathered}
$$

Based on the structure of $\mathscr{F}_{m}$ in Equation (7) and the partition of $Q_{\underline{G}}$ in Equation (15), we make

$$
\left(\begin{array}{c}
\Gamma_{1}^{H} \mathscr{L}_{m} \Gamma_{1} \\
\Gamma_{2}^{H} \mathscr{F}_{m} \Gamma_{1}
\end{array}\right)=R_{\underline{G}}\left(\begin{array}{c}
\operatorname{diag}\left(\tilde{\theta}_{1}, \ldots, \tilde{\theta}_{k}\right) \\
\alpha_{1}, \ldots, \alpha_{k}
\end{array}\right) \Theta_{1}^{-1}
$$

If we denote

$$
\begin{aligned}
& {\left[P_{0}, \tilde{W}_{1}\right]^{\text {new }}=\left[\mathscr{V}_{m},\left[P_{m-1}, \tilde{W}_{m}\right]\right] \Gamma_{2}, \quad \mathscr{V}_{1}^{\text {new }}=\mathscr{V}_{m} \Gamma_{1},} \\
& \mathscr{L}_{1}^{\text {new }}=\Gamma_{1}^{H} \mathscr{L}_{m} \Gamma_{1}, \\
& \mathbb{H}_{1}^{\text {new }}=\Gamma_{2}^{H} \mathscr{F}_{m} \Gamma_{1}, \quad \mathscr{F}_{1}^{\text {new }}=\left[\begin{array}{c}
\mathscr{L}_{1}^{\text {new }} \\
\mathbb{H}_{1}^{\text {new }}
\end{array}\right]
\end{aligned}
$$


and substitute Equation (23) into Equation (22), Equation $\sqrt{19})$ is obtained.

- Show $R_{0}=\left[\mathscr{V}_{1}^{\text {new }},\left[P_{0}, \tilde{W}_{1}\right]^{\text {new }}\right] \Lambda_{1}^{\text {new }}$ :

At restart we have

$$
\begin{aligned}
R_{0} & =R_{m} \\
& =\left[\mathscr{V}_{m},\left[P_{m-1}, \tilde{W}_{m}\right]\right] R_{L S_{m}} \\
& =\left[\mathscr{V}_{m},\left[P_{m-1}, \tilde{W}_{m}\right]\right] Q_{\underline{G}} \Theta_{2} \\
& =\left[\mathscr{V}_{1}^{\text {new }},\left[P_{0}, \tilde{W}_{1}\right]^{\text {new }}\right] \Lambda_{1}^{\text {new }} .
\end{aligned}
$$

- Show that Equality 20 holds:

Because $\left[\mathbb{W}_{1}^{\text {new }}, \mathbb{W}_{2}^{\text {new }}\right]$ is unitary, we have

$$
\left[P_{0}, \tilde{W}_{1}\right]^{\text {new }}=\left[P_{0}, \tilde{W}_{1}\right]^{\text {new }}\left[\mathbb{W}_{1}^{\text {new }} \mathbb{W}_{1}^{\text {new } H}+\mathbb{W}_{2} \mathbb{W}_{2}^{\text {new } H}\right],
$$

substituting which into Equation $(19)$ gives

$$
\begin{aligned}
A \mathscr{V}_{1}^{\text {new }} & =\left[\mathscr{V}_{1}^{\text {new }},\left[P_{0}, \tilde{W}_{1}\right]^{\text {new }}\left(\mathbb{W}_{1}^{\text {new }} \mathbb{W}_{1}^{\text {new } H}+\mathbb{W}_{2}^{\text {new }} \mathbb{W}_{2}^{\text {new } H}\right)\right]\left[\begin{array}{c}
\mathscr{L}_{1}^{\text {new }} \\
\mathbb{H}_{1}^{\text {new }}
\end{array}\right] \\
& =\mathscr{V}_{1}^{\text {new }} \mathscr{L}_{1}^{\text {new }}+\left[P_{0}, \tilde{W}_{1}\right]^{\text {new }}\left(\mathbb{W}_{1}^{\text {new }} \mathbb{W}_{1}^{\text {new } H}+\mathbb{W}_{2}^{\text {new }} \mathbb{W}_{2}^{\text {new } H}\right) \mathbb{H}_{1}^{\text {new }} \\
& =\mathscr{V}_{1}^{\text {new }} \mathscr{L}_{1}^{\text {new }}+\left[P_{0}, \tilde{W}_{1}\right]^{\text {new }} \mathbb{W}_{1}^{\text {new }} \mathbb{W}_{1}^{\text {new }} \mathbb{H}_{1}^{\text {new }}+\left[P_{0}, \tilde{W}_{1}\right]^{\text {new }} \mathbb{W}_{2}^{\text {new }} \mathbb{W}_{2}^{\text {new }} \mathbb{H}_{1}^{\text {new }} \\
& =\mathscr{V}_{1}^{\text {new }} \mathscr{L}_{1}^{\text {new }}+V_{2}^{\text {new }} \mathscr{L}_{2,:}^{\text {new }}+P_{1}^{\text {new }} G_{1}^{\text {new }} \\
& =\left[\mathscr{V}_{1}^{\text {new }}, V_{2}^{\text {new }}\right]\left[\begin{array}{l}
\mathscr{L}_{1}^{\text {new }} \\
\mathscr{L}_{2,:}^{\text {new }}
\end{array}\right]+P_{1}^{\text {new }} G_{1}^{\text {new }},
\end{aligned}
$$

which shows Equation 200 .

It is noticed that the matrix $\left[P_{0}, \tilde{W}_{1}\right]^{\text {new }}$ is treated as an integrated matrix during the computation in Proposition 2 and it is not necessary to tell the explicit forms of $P_{0}^{\text {new }}$ and $\tilde{W}_{1}^{\text {new }}$. As a matter of fact, continuing Algorithm 3 for $j=2, \ldots, m$ with the initialization proposed in Proposition 2 as shown in Steps 4 and 5 of Algorithm 2 , i.e., setting $\mathscr{V}_{2}=\mathscr{V}_{2}^{\text {new }}, \mathscr{L}_{1}=\underline{L}_{1}^{\text {new }}, P_{1}=$ $P_{1}^{\text {new }}, G_{1}=G_{1}^{\text {new }}$, we can consequently see that the block Arnoldi-like recurrence formulae of IB-BGMRES still hold for IB-BGMRES-DR. Then, an immediate corollary of Propositions 1 and 2 follows [28, Theorem 3] and reads:

Corollary 1. The subspace for IB-BGMRES-DR is a block Krylov subspace, i.e., it is a combination of Krylov subspaces after a cycle of IB-BGMRES-DR.

\subsubsection{Algorithm for restarting with deflated targeted harmonic Ritz vectors}

The glue procedure for adding the refined information of the targeted harmonic Ritz vectors at each restart of Algorithm 3 is summarized as in Algorithm 4. This glue procedure could be considered as counterparts of the implementations carried out at the Steps (4)-(7) of BGMRES-DR in [28] under the inexact-breakdown circumstances. It is remarked that in practical computation with finite precision arithmetic, it might be necessary to perform the reorthogonalization step as shown in Step 5 of Algorithm 4 to keep the orthogonality between $\left[P_{0}, \tilde{W}_{1}\right]^{\text {new }}$ and $\mathscr{V}_{1}^{\text {new }}$. As a consequence, the corresponding variables should be updated consequently as shown in Step 6 of Algorithm 4 . One can follow the proof of Proposition 2 to easily see that the initial block Arnoldi-like recurrence formulae (19) and (20) keep the same. 


\subsubsection{Right-hand sides of the least-squares problem}

For classical block-GMRES, the first residuals are in the initial search space so that the right-hand side of the least-squares problem has a special structure with non-zero entries only in its first leading block. When inexact breakdowns are handled, the right-hand side of the least-squares problem does not enjoy this property anymore and its calculation deserves some additional attention. At restart, instead of computing $R_{0}$ explicitly as $R_{0}=B-A X_{0}$, we can express it alternatively as

$$
R_{0}=\left[\mathscr{V}_{1},\left[P_{0}, \tilde{W}_{1}\right]\right] \Lambda_{1} .
$$

The necessity and motivation to update the right-hand sides of the least-squares problem at each iteration is that $R_{0}$ has components in $\left[P_{0}, \tilde{W}_{1}\right]$, those directions are not kept as is in the search space. As the method progresses, the directions of $\left[P_{0}, \tilde{W}_{1}\right]$ will contribute to the construction of $V_{j}$ or $P_{j-1}$ to expand the search space. Proposition 3 indicates how the least-squares right-hand sides can be incrementally and cheaply computed at each iteration of the block algorithm.

Proposition 3. At each iteration (see Step 5 of Algorithm 2), the new least-squares problem reads

$$
Y_{j+1}=\underset{Y \in \mathbb{C}^{n_{j+1} \times p}}{\operatorname{argmin}}\left\|\Lambda_{j+1}-\mathscr{F}_{j+1} Y\right\|_{F},
$$

with the right-hand sides being

$$
\Lambda_{j+1}=\left[\begin{array}{ll}
I_{p_{1}} & \Phi_{j+1} \\
0_{\left(n_{j+1}+p-p_{1}\right) \times p_{1}} &
\end{array}\right] \Lambda_{1},
$$

where $\Lambda_{1} \in \mathbb{C}^{\left(p_{1}+p\right) \times p}$ and $\Phi_{j+1} \in \mathbb{C}^{\left(n_{j+1}+p\right) \times p}$ can be iteratively updated as

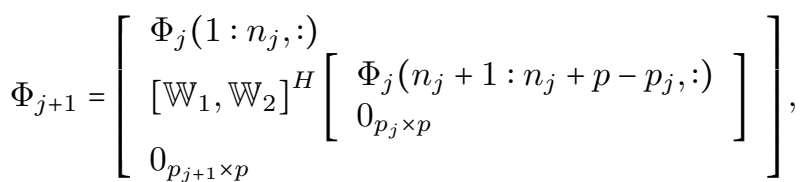

where, $\left[\mathbb{W}_{1}, \mathbb{W}_{2}\right]$ are respectively defined in Equations (8) and (10) at iteration $j$ (see Step 7 of Algorithm 4 and Algorithm 3).

Proof. The right-hand side of the least-squares problem at iteration $(j+1)$ is defined by

$$
\begin{aligned}
& \Lambda_{j+1}=\left[\mathscr{V}_{j+1},\left[P_{j}, \tilde{W}_{j+1}\right]\right]^{H} R_{0} \\
& =\left[\mathscr{V}_{j}, V_{j+1},\left[P_{j}, \tilde{W}_{j+1}\right]\right]^{H} R_{0}, \\
& =\left[\mathscr{V}_{j},\left[P_{j-1}, \tilde{W}_{j}\right] \mathbb{W}_{1},\left[P_{j-1}, \tilde{W}_{j}\right] \mathbb{W}_{2}, \tilde{W}_{j+1}\right]^{H} R_{0} \text {, using Equations } 8 \text { and }(10 \text {, } \\
& =\left[\mathscr{V}_{j},\left[P_{j-1}, \tilde{W}_{j}\right]\left[\mathbb{W}_{1}, \mathbb{W}_{2}\right], \tilde{W}_{j+1}\right]^{H} R_{0}, \\
& =\left[\mathscr{V}_{j},\left[P_{j-1}, \tilde{W}_{j}\right]\left[\mathbb{W}_{1}, \mathbb{W}_{2}\right], \tilde{W}_{j+1}\right]^{H}\left[\mathscr{V}_{1},\left[P_{0}, \tilde{W}_{1}\right]\right] \Lambda_{1}, \\
& =\left[\begin{array}{cc}
\mathscr{V}_{j}^{H} \mathscr{V}_{1} & \mathscr{V}_{j}^{H}\left[P_{0}, \tilde{W}_{1}\right] \\
{\left[V_{j+1}, P_{j}\right]^{H} \mathscr{V}_{1}} & {\left[\mathbb{W}_{1}, \mathbb{W}_{2}\right]^{H}\left[P_{j-1}, \tilde{W}_{j}\right]^{H}\left[P_{0}, \tilde{W}_{1}\right]} \\
\tilde{W}_{j+1}^{H} \mathscr{V}_{1} & \tilde{W}_{j+1}^{H}\left[P_{0}, \tilde{W}_{1}\right]
\end{array}\right] \Lambda_{1}
\end{aligned}
$$

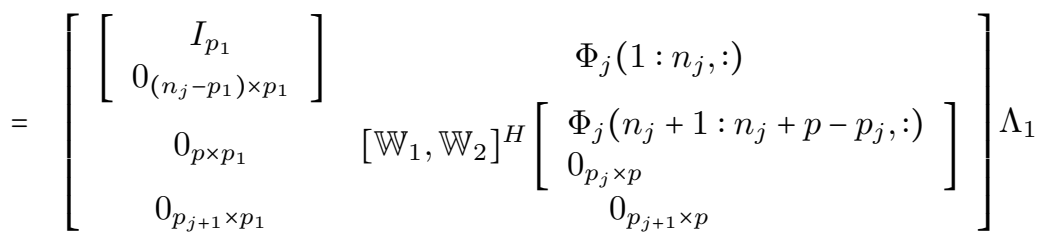


The last equality results mainly from the facts that Range $\left(\left[P_{0}, \tilde{W}_{1}\right]\right) \subset \operatorname{Range}\left(\left[\mathscr{V}_{j}, P_{j-1}, \tilde{W}_{j}\right]\right)(j=$ $1, \ldots, m), \tilde{W}_{j+1} \perp \operatorname{Range}\left(\left[P_{0}, \tilde{W}_{1}\right]\right)(j=1, \ldots, m-1)$, and that the basis $\left[\mathscr{V}_{j}, P_{j-1}, \tilde{W}_{j}\right](j=$ $1, \ldots, m)$ are orthonormal.

\subsection{The new block variant}

With the properties and discussions stated in the previous three sections, we summarize and present the new block variant-IB-BGMRES-DR as in Algorithm2. Notice that we take the same notation for IB-BGMRES-DR as that for IB-BGMRES for consistent algorithm presentation. However, it should be kept in mind that the quantities computed by IB-BGMRES-DR after the first cycle of Algorithm 2 are different from those by IB-BGMRES as shown in Algorithm 3

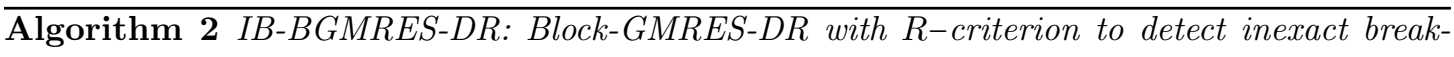
downs.

1: Start. Let the $p$ linearly independent right-hand sides be $B=\left[b^{(1)}, b^{(2)}, \ldots, b^{(p)}\right]$. Choose the maximal dimension of the underlying block approximation Krylov subspace in each cycle, $k$ the desired number of approximate targeted eigenvectors, $\varepsilon$ the targeted backward error, $X_{0}=\left[x_{0}^{(1)}, x_{0}^{(2)}, \ldots, x_{0}^{(p)}\right]$ the initial guesses. Let $r_{0}^{(i)}=b^{(i)}-A x_{0}^{(i)}, i=1, \ldots, p$. Denote $R_{0}=\left[r_{0}^{(1)}, r_{0}^{(2)}, \ldots, r_{0}^{(p)}\right]$ the initial nonsingular block residuals. The recast problems are $A\left(x^{(i)}-x_{0}^{(i)}\right)=r_{0}^{(i)}, i=1, \ldots, p$.

2: Find approximate solutions for the first cycle. Implement the first cycle of Algorithm 3 . Form the new approximate solutions $X_{m}=X_{0}+\mathscr{V}_{m} Y_{m}$. Compute the residual vectors $R_{m}=$ $B-A X_{m}=\left[\mathscr{V}_{m},\left[P_{m-1}, \tilde{W}_{m}\right]\right]\left(\Lambda_{m}-\mathscr{F}_{m} Y_{m}\right)$. Check convergence, and proceed if not satisfied.

3: Begin restart. Let $X_{0}=X_{m}$ and $R_{0}=R_{m}$. Compute the $k$ targeted eigenpairs $\left(\tilde{\theta}_{i}, \tilde{g}_{i}\right)(i=$ $1, \ldots, k)$ of the matrix $\mathscr{L}_{m}+\mathscr{L}_{m}^{-H} \mathbb{H}_{m}^{H} \mathbb{H}_{m}$. (The $\tilde{\theta}_{i}(i=1, \ldots, k)$ are harmonic Ritz values.) Then store the $\tilde{g}_{i}(i=1, \ldots, k)$ into the matrix $G$. For real matrices, it is necessary to separate $\tilde{g}_{i}(i=1, \ldots, k)$ into real and imaginary parts if complex, in order to form an $n_{m} \times k$ matrix $G$. (It may be necessary to adjust $k$ in order to make sure both parts of complex vectors are included.)

4: Implement Algorithm 4 Fulfill the initialization of Algorithm 3 at each restart with refined information associated with the $k$ targeted harmonic Ritz vectors $G$.

5: Implement Algorithm 3 for $j=2, \ldots, m$. Complete the remaining iterations of a new cycle of Algorithm 3 after the initialization with Algorithm 4 and the update of the least-squares right-hand sides described in Proposition 3

6: Form the approximate solutions. Form the new approximate solutions $X_{m}=X_{0}+\mathscr{V}_{m} Y_{m}$. Compute the residual vectors $R_{m}=B-A X_{m}=\left[\mathscr{V}_{m},\left[P_{m-1}, \tilde{W}_{m}\right]\right]\left(\Lambda_{m}-\mathscr{F}_{m} Y_{m}\right)$.

7: Check convergence, and restart if convergence is not achieved, i.e., go to 3 .

\subsection{Stopping criterion}

We further discuss how the threshold parameter $\epsilon^{(R)}$ used to detect the inexact breakdowns should be related to the final targeted backward error of the individual linear systems. The normwise backward error associated with an approximate solution $x$ with perturbation on one right-hand side $b$ is defined as follows

Definition 2. For the solution of a linear system, the normwise backward error associated with 
an approximate solution $x$ with perturbation on one right-hand side $b$ is defined by

$$
\begin{aligned}
\eta_{b}(x) & =\min _{\Delta b}\left\{\tau>0:\|\Delta b\|_{2} \leq \tau\|b\|_{2} \text { and } A x=b+\Delta b\right\} \\
& =\frac{\|A x-b\|_{2}}{\|b\|_{2}} .
\end{aligned}
$$

Assuming $p$ inexact breakdowns have occurred at iterations $s$ of IB-BGMRES (also IBBGMRES-DR) solving for $B=\left[b^{(1)}, b^{(2)}, \ldots, b^{(p)}\right]$, according to [33, Equation (47)], we have

$$
\left\|B-A X_{s}\right\|_{2} \leq \epsilon^{(R)} .
$$

Then the normwise backward errors $\eta_{b^{(i)}}(i=1, \ldots, p)$ associated with each column of approximate solutions $X_{s}=\left[x_{s}^{(1)}, \ldots, x_{s}^{(p)}\right]$ satisfy

$$
\eta_{b^{(i)}}\left(x_{s}^{(i)}\right)=\frac{\left\|b^{(i)}-A x_{s}^{(i)}\right\|_{2}}{\left\|b^{(i)}\right\|_{2}} \leq \frac{\left\|B-A X_{s}\right\|_{2}}{\left\|b^{(i)}\right\|_{2}} \leq \frac{\left\|B-A X_{s}\right\|_{2}}{\min _{i=1, \ldots, p}\left\|b^{(i)}\right\|_{2}} \leq \frac{\epsilon^{(R)}}{\min _{i=1, \ldots, p}\left\|b^{(i)}\right\|_{2}}
$$

due to the property of matrix 2 -norm. It follows that the choice

$$
\epsilon^{(R)}=\varepsilon \times \min _{i=1, \ldots, p}\left\|b^{(i)}\right\|_{2}
$$

ensures that all the linear systems have been solved to the target accuracy $\varepsilon$ when $p$ inexact breakdowns have been detected. We notice that this might induce some delay in the practical convergence check with wasted computational effort, but not much, as will be seen and discussed in the numerical experiments.

\section{Numerical experiments}

\subsection{Test problems and experimental setting}

In this section, we investigate the numerical features and convergence behaviors of IB-BGMRESDR on a set of representative test problems. The performance obtained by IB-BGMRES-DR is evaluated in comparison with BGMRES-DR [28] and IB-BGMRES [33] in term of number of matrix-vector products (referred to as mvps) that is often the most time consuming kernel in large calculations.

The test problems include eight matrices. For the sake of comparison with related works, the first five test matrices are taken as those of Example 1 in [28], the sixth matrix is used in [33, and the last two are used in [21]. From the point of view of convenient illustration, we briefly describe those examples. The first four matrices of size 1000 are bidiagonal with superdiagonal entries being all unity. Matrix 1 has diagonal entries $0.1,1,2,3, \ldots, 999$. Matrix 2 has diagonal entries $1,2,3, \ldots, 1000$, matrix 3 has diagonal entries $11,12,13, \ldots, 1010$, while matrix 4 has diagonal entries $10.1,10.2,10.3, \ldots, 19.9,20,21,22, \ldots, 919,920$. The fifth and sixth matrices are from oil reservoir simulation- SHERMAN4 of size 1104 and SHERMAN5 of size 3312 taken from the Harwell-Boeing collection of test matrices [10. The last two matrices Dehghani/light_in_tissue of size 29282 and HB/young1c of size 841 as respectively arising from light transport in soft tissue and aero research, are borrowed from the University of Florida Sparse Matrix Collection [9]. For those experiments, the $k$ smallest harmonic Ritz values are chosen as to select the harmonic vectors to be recycled in IB-BGMRES-DR and BGMRES-DR. The experiments have been carried out in double precision floating point arithmetic in MATLAB 7.0.4. 


\subsection{Some comparisons}

All these tests are started with the initial block guess equal to $0 \in \mathbb{C}^{n \times p}$. The right-hand sides $B=\left[b^{(1)}, b^{(2)}, \ldots, b^{(p)}\right] \in \mathbb{C}^{n \times p}$ have $p$ linearly independent columns generated with normal distribution. For BGMRES-DR, the stopping criterion used here is that the $p$ individual normwise backward error satisfies $\eta_{b^{(i)}}<\varepsilon(i=1, \ldots, p)$, or the number of matrix-vector multiplications exceeds the allowed maximal number (referred to as maxiter). For IB-BGMRES and IB-BGMRESDR, the stopping criterion used here is that all $p$ inexact breakdowns (monitored as described in Section 3.3 have occurred, or mvps exceeds maxiter. In those experiments we set the number of right-hand sides to $p=6$, the maximum dimension of the search space is $m=90$, the number of deflated harmonic Ritz vectors is $k=5$, the targeted backward error is $\varepsilon=10^{-6}$ and maxiter $=10000$ for all tests. No preconditioning is used except for the sixth example, where a right $\mathrm{ILU}(0)$ preconditioner is implemented to ensure the convergence within a reasonable amount of iterations.

On the right part of Figures 1 and 2, we display the upper bounds on the backward errors for IB-BGMRES and IB-BGMRES-DR given by Equation 27) and the largest backward error. It can be seen that the upper bound is quite sharp and deserves to be considered to design the stopping criterion especially when the norms of right-hand sides have similar magnitude. In the left part of these figures we display the convergence history of the three block methods reporting for each of them the smallest and largest backward errors among the $p$ right-hand sides. For problems where slow convergence is caused by small eigenvalues as Examples 1, 2, 5, 6, 7 and 8, deflation of small eigenvalues makes IB-BGMRES-DR more attractive and efficient. It enables a significant reduction of mvps compared to IB-BGMRES and BGMRES-DR. For easier problems such as Examples 3 and 4, the performances of IB-BGMRES, BGMRES-DR and IB-BGMRESDR are essentially similar. It can be observed that IB-BGMRES-DR essentially inherits the nice numerical features of its two ascendent methods namely IB-BGMRES and BGMRES-DR.

The mvps required and the attained accuracy in terms of the maximum and minimum of the $p$ individual normwise backward errors associated with the initial right-hand sides $B$ are reported in Table 1 where the symbol "-" indicates no convergence. It can be observed that the minimum components of the $p$ individual normwise backward errors attained by BGMRES-DR are smaller than their counterparts obtained by IB-BGMRES and IB-BGMRES-DR in most cases. This illustrates that some computational resources are wasted due to the none detection of partial convergence, that is BGMRES-DR does continue to enlarge the space with constant dimension at each step and update all the approximated solutions including those that have converged. This is the main feature of the IB-BGMRES-DR and IB-BGMRES variants that ensure that all the solutions converge to the same accuracy.

In order to illustrate the benefit of using block variants in linear Krylov solvers, we present numerical experiments performed with regular single GMRES $(m)$ [35] and GMRES-DR $(m, k)$ [27] for solving in sequence the same set of right-hand sides. The mvps displayed are cumulated over the $p$ solutions using the same experimental setting in term of space dimension and stopping criterion, i.e., $\eta_{b^{(i)}}<\varepsilon(i=1, \ldots, p)$, or individual mvps exceeds maxiter $(p=6, m=90, k=$ $5, \varepsilon=10^{-6}$, and maxiter $\left.=10000\right)$. The results are reported in Table 2, and they are most of the time significantly better for the block variants and only marginally worse on two examples (where it could be argued that the computer science benefit of BLAS-3 type operation can compensate the small numerical penalty). In particular, we can see that the block variants perform more efficiently than regular single vector counterparts for Examples 1, 2, 5, 6; for Examples 3, 4, all the solvers perform similarly. For Examples 7 and 8, BGMRES-DR is outperformed by its single vector counterpart GMRES-DR. In these latter two examples, IB-BGMRES-DR performs much better than BGMRES-DR, having almost the same mvps as GMRES-DR on Example 7 while 


\begin{tabular}{|c|c|c|c|c|}
\hline \multirow{2}{*}{ Example } & \multirow{2}{*}{ Method } & \multirow{2}{*}{ mvps } & \multicolumn{2}{|c|}{$\eta_{b^{(i)}}(i=1, \ldots, p)$} \\
\hline & & & $\min$ & $\max$ \\
\hline \multirow{3}{*}{1} & IB-BGMRES & 1344 & $2.23 \mathrm{e}-7$ & $5.64 \mathrm{e}-7$ \\
\hline & BGMRES-DR & 892 & $2.74 \mathrm{e}-7$ & $9.13 \mathrm{e}-7$ \\
\hline & IB-BGMRES-DR & 588 & $6.86 \mathrm{e}-7$ & $7.83 \mathrm{e}-7$ \\
\hline \multirow{3}{*}{2} & IB-BGMRES & 788 & $4.62 \mathrm{e}-7$ & $6.98 \mathrm{e}-7$ \\
\hline & BGMRES-DR & 667 & $2.96 \mathrm{e}-7$ & $9.04 \mathrm{e}-7$ \\
\hline & IB-BGMRES-DR & 538 & $7.31 \mathrm{e}-7$ & $8.53 \mathrm{e}-7$ \\
\hline \multirow{3}{*}{3} & IB-BGMRES & 372 & $6.55 \mathrm{e}-7$ & $7.85 \mathrm{e}-7$ \\
\hline & BGMRES-DR & 341 & $6.36 \mathrm{e}-7$ & $9.34 \mathrm{e}-7$ \\
\hline & IB-BGMRES-DR & 335 & $6.59 \mathrm{e}-7$ & $8.38 \mathrm{e}-7$ \\
\hline \multirow{3}{*}{4} & IB-BGMRES & 446 & $5.90 \mathrm{e}-7$ & $8.78 \mathrm{e}-7$ \\
\hline & BGMRES-DR & 447 & $6.53 \mathrm{e}-7$ & $9.18 \mathrm{e}-7$ \\
\hline & IB-BGMRES-DR & 440 & $8.18 \mathrm{e}-7$ & $9.11 \mathrm{e}-7$ \\
\hline \multirow{3}{*}{5} & IB-BGMRES & 617 & $3.93 \mathrm{e}-7$ & $9.03 \mathrm{e}-7$ \\
\hline & BGMRES-DR & 474 & $1.27 \mathrm{e}-7$ & $9.48 \mathrm{e}-7$ \\
\hline & IB-BGMRES-DR & 386 & $6.50 \mathrm{e}-7$ & $9.08 \mathrm{e}-7$ \\
\hline \multirow{3}{*}{6} & IB-BGMRES & 357 & $2.24 \mathrm{e}-7$ & $5.36 \mathrm{e}-7$ \\
\hline & BGMRES-DR & 294 & $2.71 \mathrm{e}-8$ & $9.97 \mathrm{e}-7$ \\
\hline & IB-BGMRES-DR & 248 & $6.01 \mathrm{e}-7$ & $7.54 \mathrm{e}-7$ \\
\hline \multirow{3}{*}{7} & IB-BGMRES & 3291 & $4.51 \mathrm{e}-7$ & $7.97 \mathrm{e}-7$ \\
\hline & BGMRES-DR & 3090 & $1.15 \mathrm{e}-7$ & $9.85 \mathrm{e}-7$ \\
\hline & IB-BGMRES-DR & 2104 & $8.32 \mathrm{e}-7$ & $9.55 \mathrm{e}-7$ \\
\hline \multirow{3}{*}{8} & IB-BGMRES & - & $7.01 \mathrm{e}-5$ & $5.19 \mathrm{e}-4$ \\
\hline & BGMRES-DR & 4426 & $2.84 \mathrm{e}-7$ & $9.90 \mathrm{e}-7$ \\
\hline & IB-BGMRES-DR & 2202 & $5.40 \mathrm{e}-7$ & $7.63 \mathrm{e}-7$ \\
\hline
\end{tabular}

Table 1: Numerical behavior of IB-BGMRES, BGMRES-DR and IB-BGMRES-DR with $\varepsilon=10^{-6}$. 
much less mvps than GMRES-DR on Example 8.

\begin{tabular}{|c|r|r|r|r|r|}
\hline Example & GMRES & GMRES-DR & IB-BGMRES & BGMRES-DR & IB-BGMRES-DR \\
\hline 1 & 2536 & 1077 & 1344 & 892 & $\mathbf{5 8 8}$ \\
\hline 2 & 1069 & 856 & 788 & 667 & $\mathbf{5 3 8}$ \\
\hline 3 & 378 & 378 & 372 & 341 & $\mathbf{3 3 5}$ \\
\hline 4 & $\mathbf{4 1 2}$ & $\mathbf{4 1 2}$ & 446 & 447 & 440 \\
\hline 5 & 845 & 694 & 617 & 474 & $\mathbf{3 8 6}$ \\
\hline 6 & 464 & 464 & 357 & 294 & $\mathbf{2 4 8}$ \\
\hline 7 & 3154 & $\mathbf{2 0 0 3}$ & 3291 & 3090 & 2104 \\
\hline 8 & 10643 & 3110 & - & 4426 & $\mathbf{2 2 0 2}$ \\
\hline
\end{tabular}

Table 2: Number of mvps for regular GMRES, GMRES-DR, IB-BGMRES, BGMRES-DR and IBBGMRES-DR with $\varepsilon=10^{-6}$.

\subsection{Influence of the number of right-hand sides}

In this section, we investigate the effect of the number of right-hand sides on the relative performance of the block solvers. In all the tables, we vary the number of right-hand sides $p=6,12,18,24$. First in Table 3 (Table 4) we consider $m=90$ (respectively $m=200$ ) for the maximal dimension of the search space. For the block GMRES variant, when $p$ is increased the degree of the polynomial in $A$ used to build the Krylov space decreases. For instance with $p=24$, the degree is less than 4 which can explain why some block variants do not succeed to converge. When the value of $n$ is increased to 200, the polynomial degree can be increased and the robustness of the block GMRES variant increases as well.

In order to further illustrate the effect of the polynomial degree on the block Krylov solver robustness we report in Table 5 numerical results where we attempt to keep constant this polynomial degree to 15 when the number of right-hand sides is varied. Consequently we set $m=15 \times p$ and only select the largest test examples so that the maximum size of the search space remains a small fraction of the problem dimension. It can be observed that the numerical performance of all block GMRES improves. For $p=24$, regular GMRES and GMRES-DR never perform restart so that their numerical behaviours are identical.

\subsection{Inexact breakdowns versus targeted accuracy}

In this section we illustrate the benefit of the tight coupling between the inexact breakdown detection and the targeted accuracy for the solution. In Figure 3, we display the convergence history of IB-BGMRES-DR for different values of $\varepsilon$ for the largest and smallest of the $p$ backward errors. Because the inexact breakdown threshold is related to $\varepsilon$ by Equation (28), the inexact breakdown strategy will act earlier for larger values of $\varepsilon$. For instance it might be observed that the curves overlap until the first inexact breakdown is detected for $\varepsilon=10^{-6}$, then the ones associated with $\varepsilon=10^{-8}$ and $10^{-10}$ still overlap while the other one exhibits a faster decay to its target $10^{-8}$. This characterizes the nice feature of the inexact breakdown mechanism that enables to focus on what remains to be converged and then reduce the computational cost to achieve convergence. 


\begin{tabular}{|c|c|c|c|c|c|c|c|c|c|}
\hline$p$ & $\begin{array}{l}\text { Example } \\
\text { Method }\end{array}$ & 1 & 2 & 3 & 4 & 5 & 6 & 7 & 8 \\
\hline \multirow{5}{*}{6} & GMRES & 2536 & 1069 & 378 & 412 & 845 & 464 & 3154 & - \\
\hline & GMRES-DR & 1077 & 856 & 378 & 412 & 694 & 464 & 2003 & 3110 \\
\hline & IB-BGMRES & 1344 & 788 & 372 & 446 & 617 & 357 & 3291 & - \\
\hline & BGMRES-DR & 892 & 667 & 341 & 447 & 474 & 294 & 3090 & 4426 \\
\hline & IB-BGMRES-DR & 588 & 538 & 335 & 440 & 386 & 248 & 2104 & 2202 \\
\hline \multirow{5}{*}{12} & GMRES & 4855 & 2132 & 753 & 825 & 1696 & 927 & 6343 & - \\
\hline & GMRES-DR & 2141 & 1711 & 753 & 825 & 1404 & 927 & 4003 & 6225 \\
\hline & IB-BGMRES & 2427 & 1556 & 790 & 1091 & 1182 & 713 & 7320 & - \\
\hline & BGMRES-DR & 2324 & 1784 & 820 & 1139 & 1209 & 901 & - & - \\
\hline & IB-BGMRES-DR & 1098 & 1074 & 694 & 1062 & 843 & 442 & 5823 & 4478 \\
\hline \multirow{5}{*}{18} & GMRES & 7573 & 3235 & 1135 & 1238 & 2556 & 1393 & 9641 & - \\
\hline & GMRES-DR & 3204 & 2553 & 1135 & 1238 & 2117 & 1393 & 6029 & 9332 \\
\hline & IB-BGMRES & 3048 & 2322 & 1278 & 1902 & 1913 & 1116 & - & - \\
\hline & BGMRES-DR & 5529 & 4207 & 1536 & 2587 & 2355 & - & - & - \\
\hline & IB-BGMRES-DR & 1822 & 1687 & 1178 & 2058 & 1579 & 661 & - & 6794 \\
\hline \multirow{5}{*}{24} & GMRES & - & 4332 & 1516 & 1648 & 3367 & 1859 & - & - \\
\hline & GMRES-DR & 4277 & 3408 & 1516 & 1648 & 2824 & 1859 & 8015 & - \\
\hline & IB-BGMRES & 4542 & 3244 & 1902 & 3589 & 2961 & 1589 & - & - \\
\hline & BGMRES-DR & & 6963 & 2375 & 4531 & 3741 & - & - & - \\
\hline & IB-BGMRES-DR & 2402 & 2312 & 1648 & 3349 & 2473 & 846 & - & 8744 \\
\hline
\end{tabular}

Table 3: Total number of mvps for different numbers of right-hand sides with $m=90$. 

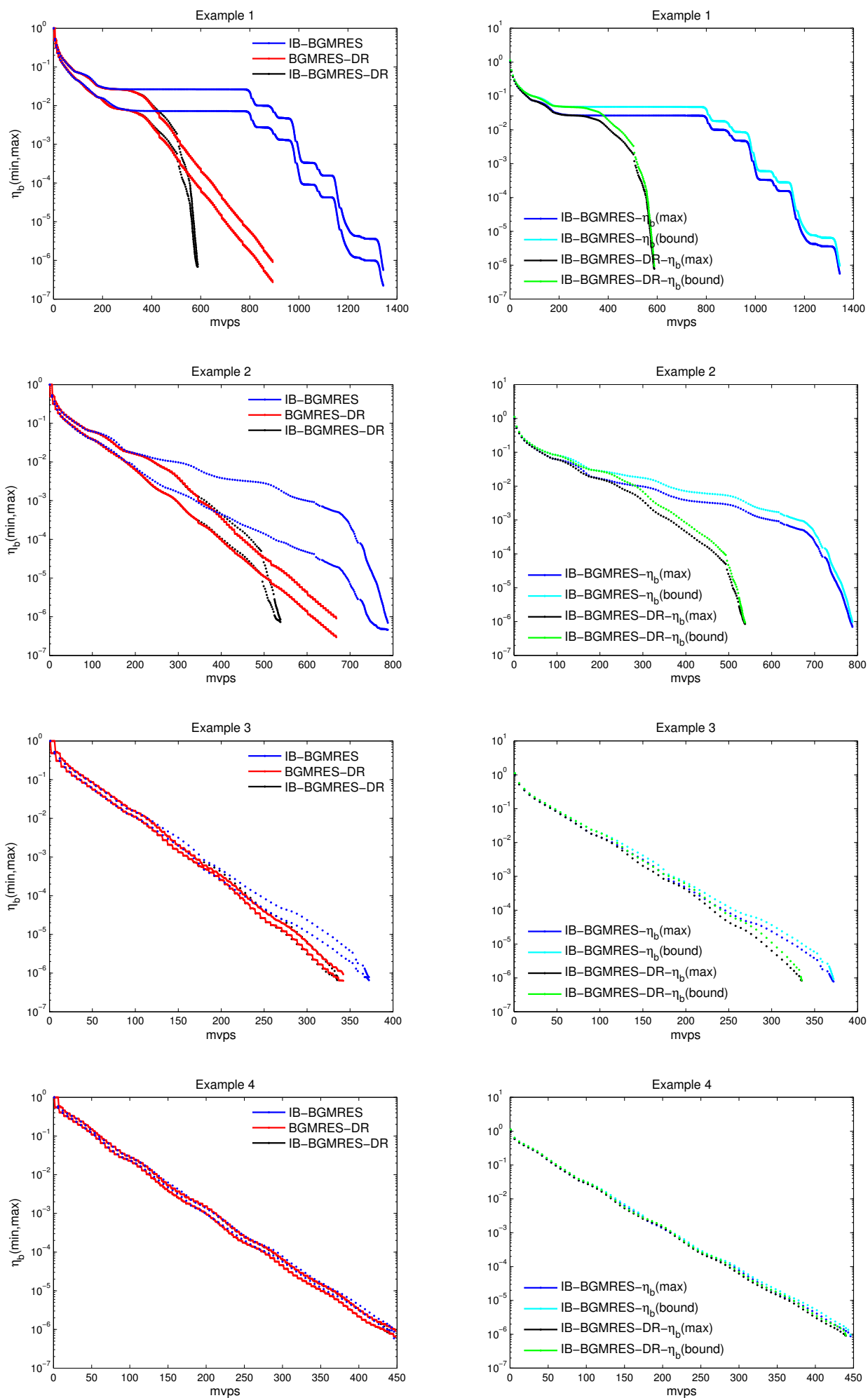

Figure 1: Convergence histories for the first four examples. Left: convergence histories of the largest/smallest $\eta_{b^{(i)}}$ at each mvps. Right: upper bounds of $\eta_{b^{(i)}}$ and $\max \left(\eta_{b^{(i)}}\right)$ at each mvps. $\mathrm{RR} \mathrm{n}^{\circ} 8503$ 

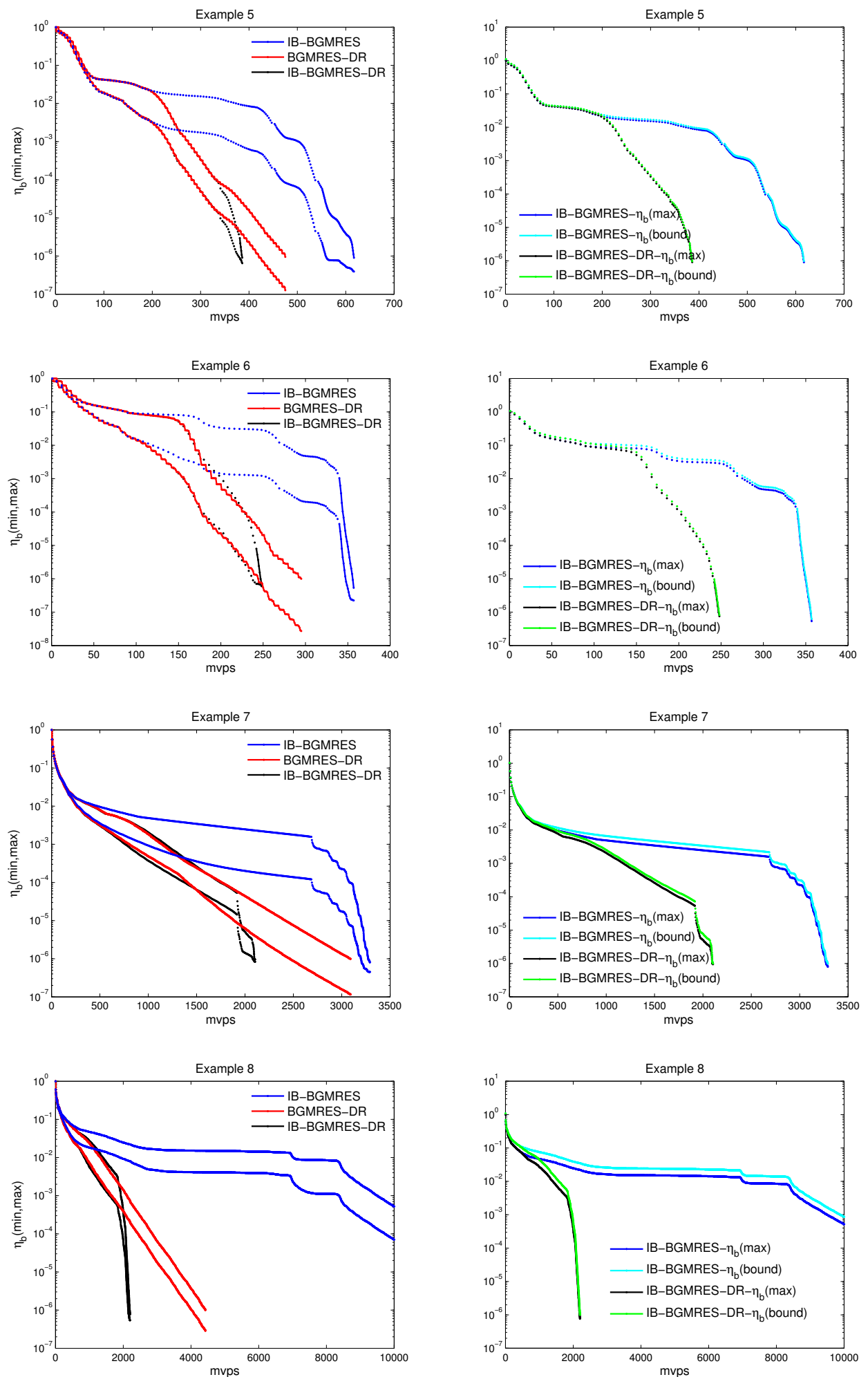

Figure 2: Convergence histories for the last four examples. Left: convergence histories of the largest/smallest $\eta_{b^{(i)}}$ at each mvps. Right: upper bounds of $\eta_{b^{(i)}}$ and $\max \left(\eta_{b^{(i)}}\right)$ at each mvps. 


\begin{tabular}{|c|c|c|c|c|c|c|c|c|c|}
\hline$p$ & $\begin{array}{l}\text { Example } \\
\text { Method }\end{array}$ & 1 & 2 & 3 & 4 & 5 & 6 & 7 & 8 \\
\hline \multirow{5}{*}{6} & GMRES & 1062 & 849 & 378 & 412 & 660 & 464 & 2428 & 4012 \\
\hline & GMRES-DR & 1062 & 849 & 378 & 412 & 660 & 464 & 1977 & 2211 \\
\hline & IB-BGMRES & 759 & 596 & 327 & 410 & 367 & 184 & 2230 & 2738 \\
\hline & BGMRES-DR & 677 & 582 & 328 & 417 & 371 & 225 & 2004 & 1645 \\
\hline & IB-BGMRES-DR & 516 & 473 & 315 & 410 & 316 & 184 & 1524 & 1361 \\
\hline \multirow{5}{*}{12} & GMRES & 2112 & 1698 & 753 & 825 & 1330 & 927 & 4829 & 8126 \\
\hline & GMRES-DR & 2112 & 1698 & 753 & 825 & 1330 & 927 & 3951 & 4435 \\
\hline & IB-BGMRES & 1183 & 1059 & 645 & 850 & 703 & 368 & 4481 & 4663 \\
\hline & BGMRES-DR & 1219 & 981 & 627 & 853 & 699 & 393 & 5857 & 4474 \\
\hline & IB-BGMRES-DR & 829 & 797 & 573 & 828 & 568 & 345 & 3342 & 2465 \\
\hline \multirow{5}{*}{18} & GMRES & 3162 & 2533 & 1135 & 1238 & 2004 & 1393 & 7335 & 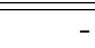 \\
\hline & GMRES-DR & 3162 & 2533 & 1135 & 1238 & 2004 & 1393 & 5950 & 6650 \\
\hline & IB-BGMRES & 1651 & 1469 & 947 & 1341 & 963 & 550 & 6944 & 8128 \\
\hline & BGMRES-DR & 2667 & 1756 & 981 & 1453 & 1170 & 735 & - & 7580 \\
\hline & IB-BGMRES-DR & 1189 & 1120 & 850 & 1316 & 855 & 475 & 6034 & 3527 \\
\hline \multirow{5}{*}{24} & GMRES & 4220 & 3380 & 1516 & 1648 & 2671 & 1859 & 9670 & - \\
\hline & GMRES-DR & 4220 & 3380 & 1516 & 1648 & 2671 & 1859 & 7907 & 8865 \\
\hline & IB-BGMRES & 2170 & 1930 & 1217 & 1961 & 1386 & 731 & - & - \\
\hline & BGMRES-DR & 3500 & 2856 & 1374 & 2103 & 1759 & 960 & - & - \\
\hline & IB-BGMRES-DR & 1513 & 1500 & 1115 & 1905 & 1221 & 591 & 8731 & 4254 \\
\hline
\end{tabular}

Table 4: Total number of mvps for different numbers of right-hand sides with $m=200$.
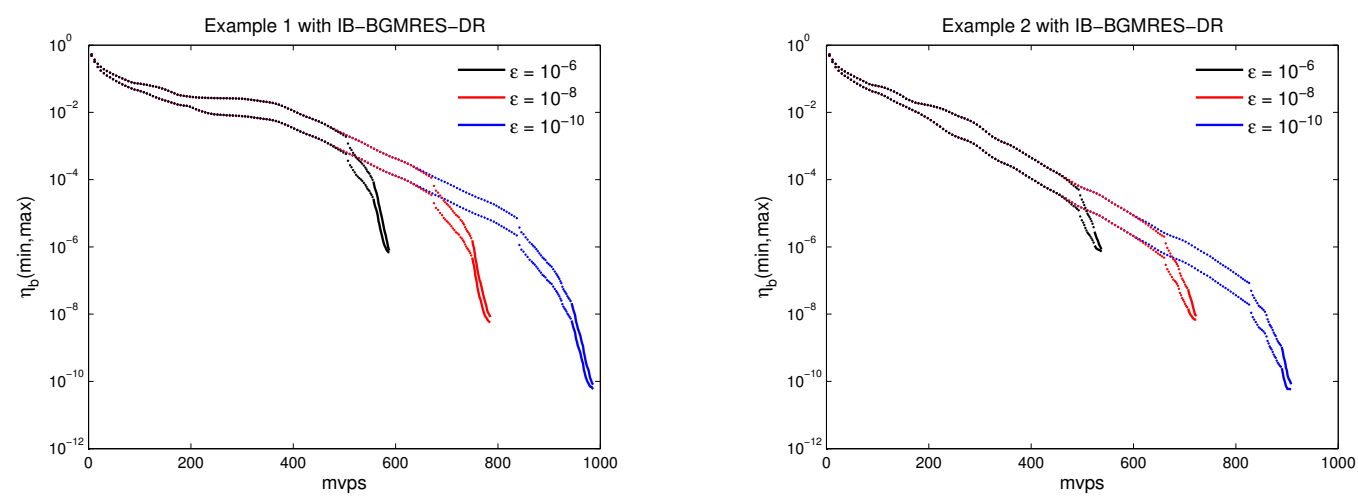

Figure 3: Convergence history of IB-GMRES-DR when the targeted accuracy is varied. 


\begin{tabular}{|c|l|r|r|r|}
\hline$p$ & $\begin{array}{l}\text { Example } \\
\text { Method }\end{array}$ & 5 & 6 & 7 \\
\hline \multirow{4}{*}{6} & GMRES & 845 & 464 & 3154 \\
\cline { 2 - 5 } & GMRES-DR & 694 & 464 & $\mathbf{2 0 0 3}$ \\
\cline { 2 - 5 } & IB-BGMRES & 617 & 357 & 3291 \\
\cline { 2 - 5 } & BGMRES-DR & 474 & 294 & 3090 \\
\cline { 2 - 5 } & IB-BGMRES-DR & $\mathbf{3 8 6}$ & $\mathbf{2 4 8}$ & 2104 \\
\hline \hline \multirow{4}{*}{12} & GMRES & 1330 & 927 & 4845 \\
\cline { 2 - 5 } & GMRES-DR & 1330 & 927 & 3946 \\
\cline { 2 - 5 } & IB-BGMRES & 673 & 406 & 4787 \\
\cline { 2 - 5 } & BGMRES-DR & 719 & 481 & 7567 \\
\cline { 2 - 5 } & IB-BGMRES-DR & $\mathbf{5 8 9}$ & $\mathbf{3 4 4}$ & $\mathbf{3 5 7 6}$ \\
\hline \hline \multirow{4}{*}{18} & GMRES & 2004 & 1393 & 6270 \\
\cline { 2 - 5 } & GMRES-DR & 2004 & 1393 & 5963 \\
\cline { 2 - 5 } & IB-BGMRES & 831 & 454 & 5843 \\
\cline { 2 - 5 } & BGMRES-DR & 981 & 507 & - \\
\cline { 2 - 5 } & IB-BGMRES-DR & $\mathbf{7 4 4}$ & $\mathbf{4 2 7}$ & $\mathbf{4 9 6 3}$ \\
\hline \hline \multirow{3}{*}{24} & GMRES & 2671 & 1859 & 7845 \\
\cline { 2 - 5 } & GMRES-DR & 2671 & 1859 & 7845 \\
\cline { 2 - 5 } & IB-BGMRES & 941 & 495 & 7149 \\
\cline { 2 - 5 } & BGMRES-DR & 1241 & 600 & - \\
\cline { 2 - 5 } & IB-BGMRES-DR & $\mathbf{8 5 2}$ & $\mathbf{4 3 7}$ & $\mathbf{6 1 1 4}$ \\
\hline
\end{tabular}

Table 5: Examples 5, 6 and 7: Comparison results of "iso-polynomial degree" experiments for different numbers of right-hand sides with $m=15 \times p$. 


\subsection{Further improvement}

The selection of the restart parameter for $\operatorname{GMRES}(m)$ depends mostly on the specific application context and on the affordable memory. Recently, it has been surprisingly noticed that small values of the restart parameter actually yield convergence in fewer iterations than larger values for some problems (see, e.g. [3, 4, 11, 12] and related papers, e.g. 41]). In [3] the authors proposed a simple strategy for varying the restart parameter of $\operatorname{GMRES}(m)$ with a negligible amount of overhead to accelerate convergence in terms of the time to solution by disrupting repetitive behaviors of the GMRES $(m)$ residual vectors at the end of each restart cycle.

Corresponding to the selection of the restart parameter for $\operatorname{GMRES}(m)$, the selection of the maximal dimension $n$ of the underlying block approximation Krylov subspace in each cycle of IB-BGMRES-DR is examined in this section. The strategy employed in 3 can be extended in a straightfordward way to adjust $n$ the maximal dimension of the block Krylov subspace in each cycle in IB-BGMRES-DR. Instead of using the residual norm as in [3] we use the bounds on the 2-norm of the residual block. The calculation of $n$ is described in Algorithm 5 that involves a negligible extra computational effort.

As shown in Table 6, for small values of $n$, the adjusting strategy does help to further reduce mvps required by IB-BGMRES-DR. While for larger ones, the strategy seems not to help much for some cases. However, from the point of view of the goal of restarting as a means of reducing computational and storage costs, this adjusting strategy could be recommended, especially when memory is a concern. It should be noted that this strategy could be also extended to other block

\begin{tabular}{|l|l|l|l|l|l|l|l|l|}
\hline $\begin{array}{l}\text { Example } \\
\text { Method }\end{array}$ & 1 & 2 & 3 & 4 & 5 & 6 & 7 & 8 \\
\hline IB-BGMRES-DR(30) & 1043 & 917 & 508 & 728 & 709 & 304 & 5330 & 5064 \\
\hline$\alpha$ IB-BGMRES-DR $(30,15)$ & $\mathbf{6 7 1}$ & $\mathbf{5 8 1}$ & $\mathbf{3 6 4}$ & $\mathbf{4 7 6}$ & $\mathbf{4 7 6}$ & $\mathbf{2 7 0}$ & $\mathbf{1 9 5 4}$ & 4691 \\
\hline \hline IB-BGMRES-DR(60) & 688 & 615 & 370 & $\mathbf{4 9 1}$ & $\mathbf{4 5 1}$ & $\mathbf{2 7 8}$ & 2705 & 2762 \\
\hline$\alpha$ IB-BGMRES-DR $(60,15)$ & $\mathbf{6 3 9}$ & $\mathbf{5 9 9}$ & $\mathbf{3 6 6}$ & 545 & 489 & 286 & $\mathbf{1 6 9 1}$ & $\mathbf{2 5 1 6}$ \\
\hline \hline IB-BGMRES-DR $(90)$ & $\mathbf{5 8 8}$ & $\mathbf{5 3 8}$ & 335 & 440 & 386 & 248 & 2104 & $\mathbf{2 2 0 2}$ \\
\hline$\alpha$ IB-BGMRES-DR $(90,15)$ & 603 & 545 & 335 & 440 & 387 & 248 & $\mathbf{1 6 7 3}$ & 2359 \\
\hline
\end{tabular}

Table 6: Comparison results for varying the maximal dimension of the underlying block approximation Krylov subspace in each cycle with $\varepsilon=10^{-6}$ in terms of mvps.

variants of $\operatorname{GMRES}(m)$, however this is out of the scope of this paper and we do not investigate it further.

\section{Concluding remarks}

In this paper we combine the numerical feature of BGMRES-DR and IB-BGMRES to develop a new block variant-IB-BGMRES-DR for systems of linear equations with multiple right-hand sides given simultaneously. We derive a restarting strategy that preserves the Arnoldi-like relation while ensuring that both the residuals and some spectral information are in the space at restart when inexact breakdown occured. Not addressed in 33, we also show how the right-hand sides of the least-squares problems can be incrementally updated after the first restart of IB-BGMRES and IB-BGMRES-DR. Through extensive numerical experiments we illustrate that IB-BGMRESDR inherits from the nice numerical features of its two ascendent methods. In particular, we indicate how the varying restart strategy of regular GMRES can be extended to this new block 
variant. Finally, although not derived in this paper, a version of the proposed algorithm with flexible preconditioning can be developed using similar techniques as those presented in [16].

\section{Acknowlegements}

The authors would like to thank Xavier Vasseur from CERFACS for reviewing a preliminary version of this manuscript. The third author wishes to thank Prof. Ting-Zhu Huang for carefully reading earlier version of this paper and is grateful to the team members in HiePACS, Inria Bordeaux-Sud-Ouest for warm hospitality.

\section{References}

[1] A. H. Baker, J. M. Dennis, and E. R. Jessup. An efficient block variant of GMRES. SIAM J. Scientific Computing, 27:1608-1626, 2006.

[2] A. H. Baker, J. M. Dennis, and E. R. Jessup. On improving linear solver performance: A block variant of GMRES. SIAM J. Scientific Computing, 27(5):1608-1628, 2006. doi: $10.1137 / 040608088$.

[3] A. H. Baker, E. R. Jessup, and Tz. V. Kolev. A simple strategy for varying the restart parameter in GMRES $(m)$. J. Comput. Appl. Math., 230:751-761, 2009.

[4] A. H. Baker, E. R. Jessup, and T. Manteuffel. A technique for accelerating the convergence of restarted GMRES. SIAM J. Matrix Analysis and Applications, 26:962-984, 2005.

[5] H. Calandra, S. Gratton, J. Langou, X. Pinel, and X. Vasseur. Flexible variants of block restarted GMRES methods with application to geophysics. SIAM J. Scientific Computing, 34(2):A714-A736, 2012.

[6] C. Le Calvez and B. Molina. Implicitly restarted and deflated GMRES. Numerical Algorithms, 21:261-285, 1999.

[7] A. Chapman and Y. Saad. Deflated and augmented Krylov subspace techniques. Numerical Linear Algebra with Applications, 4:43-66, 1997.

[8] D. Darnella, R. B. Morgan, and W. Wilcox. Deflated GMRES for systems with multiple shifts and multiple right-hand sides. Linear Algebra and its Applications, 429:2415-2434, 2008.

[9] T. A. Davis. The university of florida sparse matrix collection. NA DIGEST, 92, 1994.

[10] I. S. Duff, R. G. Grimes, and J. G. Lewis. Sparse matrix test problems. ACM Trans. Math. Softw., 15:1-14, 1989.

[11] M. Eiermann, O. G. Ernst, and O. Schneider. Analysis of acceleration strategies for restarted minimum residual methods. J. Comput. Appl. Math., 123:261-292, 2000.

[12] M. Embree. The tortoise and the hare restart GMRES. SIAM Review, 45:259-266, 2003.

[13] R. W. Freund and M. Malhotra. A block QMR algorithm for non-hermitian linear systems with multiple right-hand sides. Linear Algebra and its Applications, 254:119-157, 1997. 
[14] A. Gaul, M. H. Gutknecht, J. Liesen, and R. Nabben. A framework for delfated and augmented Krylov subpace methods. SIAM J. Matrix Analysis and Applications, 34(2):495$518,2013$.

[15] L. Giraud, S. Gratton, and E. Martin. Incremental spectral preconditioners for sequences of linear systems. Applied Numerical Mathematics, 57(11-12):1164-1180, 2007.

[16] L. Giraud, S. Gratton, X. Pinel, and X. Vasseur. Flexible GMRES with deflated restarting. SIAM J. Scientific Computing, 32:1858-1878, 2010.

[17] G. Gu and Z. Cao. A block GMRES method augmented with eigenvectors. Applied Mathematics and Computation, 121(2-3):271-289, 2001.

[18] M. H. Gutknecht. Block Krylov space methods for linear systems with multiple right-hand sides: an introduction. In I. S. Duff A. H. Siddiqi and O. Christensen, editors, Modern Mathematical Models, Methods and Algorithms for Real World Systems, India, 2006. New Delhi.

[19] M. H. Gutknecht. Deflated and augmented Krylov subspace methods: a framework for deflated BiCG and related solvers. SIAM J. Matrix Analysis and Applications, 2014. to appear.

[20] K. Jbilou, A. Messaoudi, and H. Sadok. Global FOM and GMRES algorithms for matrix equations. Applied Numerical Mathematics, 31(1):49-63, 1999.

[21] Y.-F. Jing, T.-Z. Huang, Y. Zhang, L. Li, G.-H Cheng, Z.-G. Ren, Y. Duan, T. Sogabe, and B. Carpentieri. Lanczos-type variants of the cocr method for complex nonsymmetric linear systems. J. Comp. Phys., 228:6376-6394, 2009.

[22] J. Langou. Iterative methods for solving linear systems with multiple right-hand sides. Ph.D. Dissertation TH/PA/03/24, CERFACS, Toulouse, France, 2003.

[23] G. Li. A block variant of the GMRES method on massively parallel processors. Parallel Computing, 23:1005-1019, 1997.

[24] H.-L. Liu and B.-J. Zhong. Simpler block GMRES for nonsymmetric systems with multiple right hand sides. Electronic Transactions on Numerical Analysis, 30:1-9, 2008.

[25] R. B. Morgan. Computing interior eigenvalues of large matrices. Linear Algebra and its Applications, 154/156:289-309, 1991.

[26] R. B. Morgan. A restarted GMRES method augmented with eigenvectors. SIAM J. Matrix Analysis and Applications, 16:1154-1171, 1995.

[27] R. B. Morgan. GMRES with deflated restarting. SIAM J. Scientific Computing, 24(1):20-37, 2002.

[28] R. B. Morgan. Restarted block GMRES with deflation of eigenvalues. Applied Numerical Mathematics, 54(2):222-236, 2005.

[29] R. B. Morgan and M. Zeng. Harmonic projection methods for large non-symmetric eigenvalue problems. Numerical Linear Algebra with Applications, 5:33-55, 1998.

[30] C. C. Paige, B. N. Parlett, and H. A. Van der Vorst. Approximate solutions and eigenvalue bounds from Krylov subspaces. Numerical Linear Algebra with Applications, 2:115-133, 1995 .

$\operatorname{RR} \mathrm{n}^{\circ} 8503$ 
[31] M. Parks, E. de Sturler, G. Mackey, D.D. Johnson, and S. Maiti. Recycling Krylov subspaces for sequences of linear systems. SIAM J. Scientific Computing, 28(5):1651-1674, 2006.

[32] M. Robbé and M. Sadkane. A convergence analysis of GMRES and FOM methods for sylvester equations. Numerical Algorithms, 30(1):71-89, 2002.

[33] M. Robbé and M. Sadkane. Exact and inexact breakdowns in the block GMRES method. Linear Algebra and its Applications, 419:265-285, 2006.

[34] Y. Saad. Analysis of augmented Krylov subspace methods. SIAM J. Matrix Analysis and Applications, 18(2):435-449, 1997.

[35] Y. Saad. Iterative Methods for Sparse Linear Systems. SIAM, Philadelphia, 2003. Second edition.

[36] V. Simoncini and E. Gallopoulos. An iterative method for nonsymmetric systems with multiple right-hand sides. SIAM J. Scientific Computing, 16:917-933, 1995.

[37] V. Simoncini and E. Gallopoulos. Convergence properties of block GMRES and matrix polynomials. Linear Algebra and its Applications, 247:97-119, 1996.

[38] V. Simoncini and E. Gallopoulos. A hybrid block GMRES method for nonsymmetric systems with multiple right-hand sides. J. Comput. Appl. Math., 66:457-469, 1996.

[39] G. L. G. Sleijpen and H. A. van der Vorst. A Jacobi-Davidson iteration method for linear eigenvalue problems. SIAM J. Matrix Analysis and Applications, 17:401-425, 1996.

[40] G. W. Stewart. Selected works with commentaries. In M. E. Kilmer and D. P. O'Leary, editors, Contemporary Mathematicians, London, 2010. Springer New York Dordrecht Heidelberg. First edition, XIII, 729 p. 6 illus.

[41] E. Vecharynski and J. Langou. The cycle-convergence of restarted GMRES for normal matrices is sublinear. SIAM J. Scientific Computing, 32(1):186-196, 2010.

[42] B. Vital. Étude de quelques méthodes de résolution de problèmes linéaires de grande taille sur multiprocesseur. Ph.d. dissertation, IRISA, Université de Rennes, France, 1990. 


\section{A Robbé, Sadkane Block Arnoldi with inexact breakdowns}

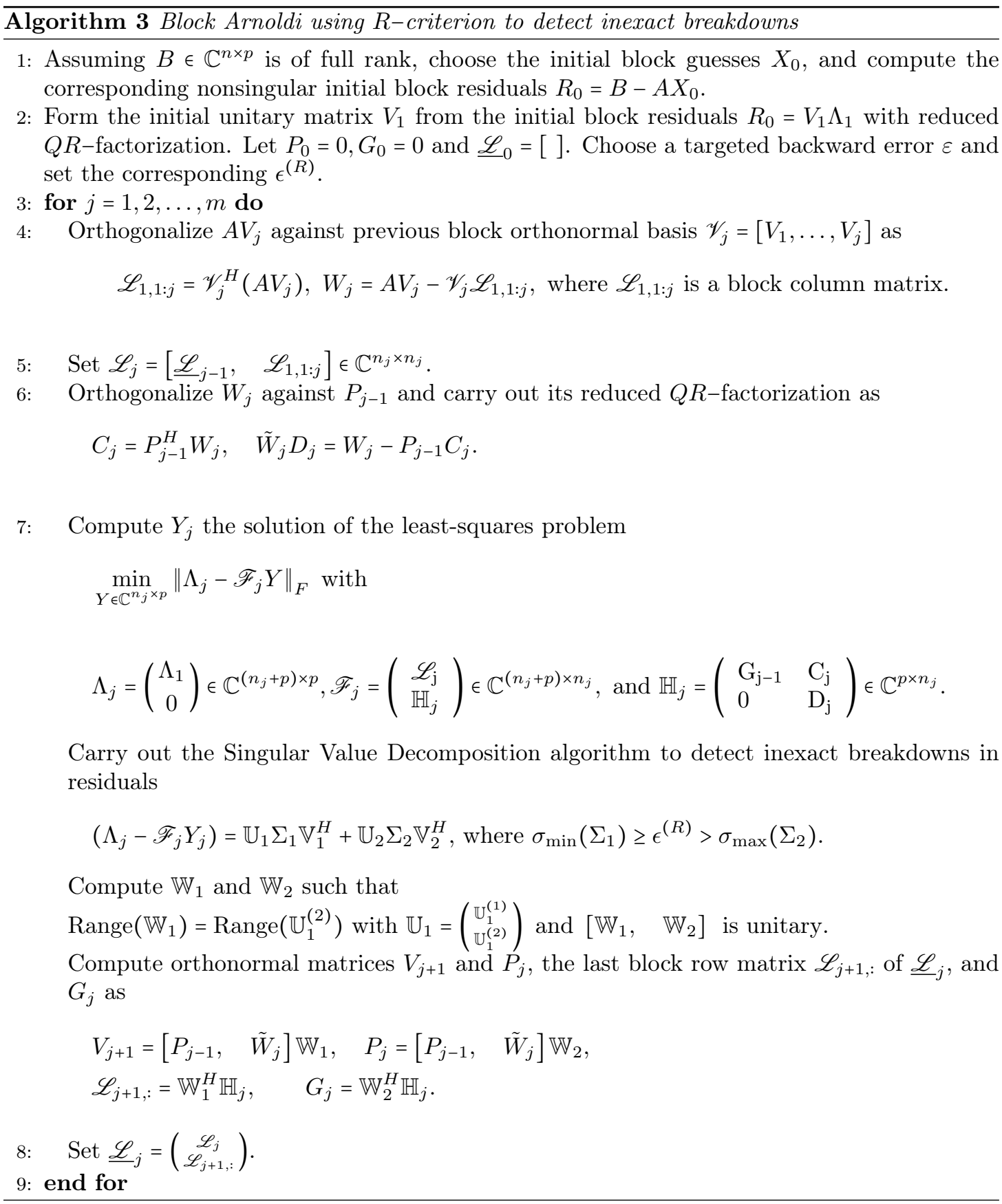

\section{B Restarting procedure with deflation}


Algorithm 4 Restarting with deflated targeted harmonic Ritz vectors

Morgan [R. B. Morgan. Restarted block GMRES with deflation of eigenvalues. Applied Numerical Mathematics, 54(2): 222-236, 2005.].

1: Compute $R_{L S_{m}}=\Lambda_{m}-\mathscr{F}_{m} Y_{m}=\left[\mathscr{V}_{m},\left[P_{m-1}, \tilde{W}_{m}\right]\right]^{H} R_{m} \in \mathbb{C}^{\left(n_{m}+p\right) \times p}$ the least-squares residuals at the $m$-th iteration of the first cycle of Algorithm 3 . Denote by $\mathscr{V}_{m} \tilde{G}$ the $k$ targeted harmonic Ritz vectors, where $\tilde{G}=\left[\tilde{g}_{1}, \ldots, \tilde{g}_{k}\right] \in \mathbb{C}^{n_{m} \times k}$.

2: Append $R_{L S_{m}}$ to $\tilde{G}$ by first adding zero rows of size $p$ to $\tilde{G}$ and then $R_{L S_{m}}$ to form a new

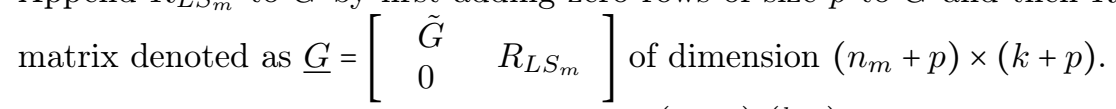

3: Form and store a unitary matrix $Q_{\underline{G}} \in \mathbb{C}^{\left(n_{m}+p\right) \times(k+p)}$ and the last $p$ columns of an upper triangular matrix $R_{\underline{G}} \in \mathbb{C}^{(k+p) \times(k+p)}$ from the reduced $Q R$-factorization of $\underline{G}$ as $\underline{G}=Q_{\underline{G}} R_{\underline{G}}$. The matrix consisting of the last $p$ columns of $R_{\underline{G}}$ is denoted as

$$
\Lambda_{1}^{\text {new }}=R_{\underline{G}}(:, k+1: k+p) \in \mathbb{C}^{(k+p) \times p} .
$$

4: Compute

$$
\begin{aligned}
& {\left[P_{0}, \tilde{W}_{1}\right]^{\text {new }}=\left[\mathscr{V}_{m},\left[P_{m-1}, \tilde{W}_{m}\right]\right] Q_{\underline{G}}(:, k+1: k+p), \quad \mathscr{V}_{1}^{\text {new }}=\mathscr{V}_{m} Q_{\underline{G}}\left(1: n_{m}, 1: k\right),} \\
& \mathscr{L}_{1}^{\text {new }}=Q_{\underline{G}}\left(1: n_{m}, 1: k\right)^{H} \mathscr{L}_{m} Q_{\underline{G}}\left(1: n_{m}, 1: k\right), \\
& \mathbb{H}_{1}^{\text {new }}=Q_{\underline{G}}(:, k+1: k+p)^{H} \mathscr{F}_{m} Q_{\underline{G}}\left(1: n_{m}, 1: k\right) .
\end{aligned}
$$

5: Reorthogonalize $\left[P_{0}, \tilde{W}_{1}\right]^{\text {new }}$ against $\mathscr{V}_{1}^{\text {new }}$ such that

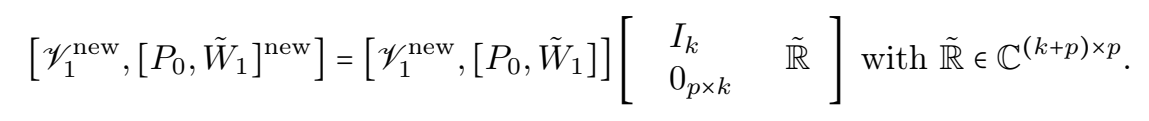

6: Compute $\mathscr{L}_{1}=\mathscr{L}_{1}^{\text {new }}+\tilde{\mathbb{R}}(1: k,:) \mathbb{H}_{1}^{\text {new }}, \mathbb{H}_{1}=\tilde{\mathbb{R}}(k+1: k+p,:) \mathbb{H}_{1}^{\text {new }}, \Lambda_{1}=\left[\begin{array}{cc}I_{k} & \tilde{\mathbb{R}} \\ 0_{p \times k}\end{array}\right] \Lambda_{1}^{\text {new }}$. Let $\mathscr{V}_{1}=\mathscr{V}_{1}^{\text {new }}$ and $n_{1}=k$. Set $\mathscr{F}_{1}=\left(\begin{array}{c}\mathscr{L}_{1} \\ \mathbb{H}_{1}\end{array}\right)$.

7: Compute $Y_{1}$ the solution of the first new least-squares problem

$$
\min _{Y \in \mathbb{C}^{n_{1} \times p}}\left\|\Lambda_{1}-\mathscr{F}_{1} Y\right\|_{F} .
$$

Carry out the Singular Value Decomposition algorithm to detect inexact breakdown in first new block residuals

$$
\left(\Lambda_{1}-\mathscr{F}_{1} Y_{1}\right)=\mathbb{U}_{1} \Sigma_{1} \mathbb{V}_{1}^{H}+\mathbb{U}_{2} \Sigma_{2} \mathbb{V}_{2}^{H}, \text { where } \sigma_{\min }\left(\Sigma_{1}\right) \geq \epsilon_{j}^{(R)}>\sigma_{\max }\left(\Sigma_{2}\right) .
$$

Compute $\mathbb{W}_{1}$ and $\mathbb{W}_{2}$ such that

$\operatorname{Range}\left(\mathbb{W}_{1}\right)=\operatorname{Range}\left(\mathbb{U}_{1}^{(2)}\right)$ with $\mathbb{U}_{1}=\left(\begin{array}{c}\mathbb{U}_{1}^{(1)} \\ \mathbb{U}_{1}^{(2)}\end{array}\right)$ and $\left[\mathbb{W}_{1}, \quad \mathbb{W}_{2}\right]$ is unitary.

Compute new orthonormal matrices $V_{2}$ and $P_{1}$, the last block row matrix $\mathscr{L}_{2 \text {,: }}$ of $\underline{L}_{1}$, and $G_{1}$ as

$$
V_{2}=\left[P_{0}, \tilde{W}_{1}\right] \mathbb{W}_{1}, P_{1}=\left[P_{0}, \tilde{W}_{1}\right] \mathbb{W}_{2}, \mathscr{L}_{2,:}=\mathbb{W}_{1}^{H} \mathbb{H}_{1}, G_{1}=\mathbb{W}_{2}^{H} \mathbb{H}_{1}
$$

8: Set $\mathscr{L}_{1}=\left(\begin{array}{c}\mathscr{L}_{1} \\ \mathscr{L}_{2,:}\end{array}\right)$ 


\section{Alpha variant of BGMRES with inexact breakdowns and deflated restarting}

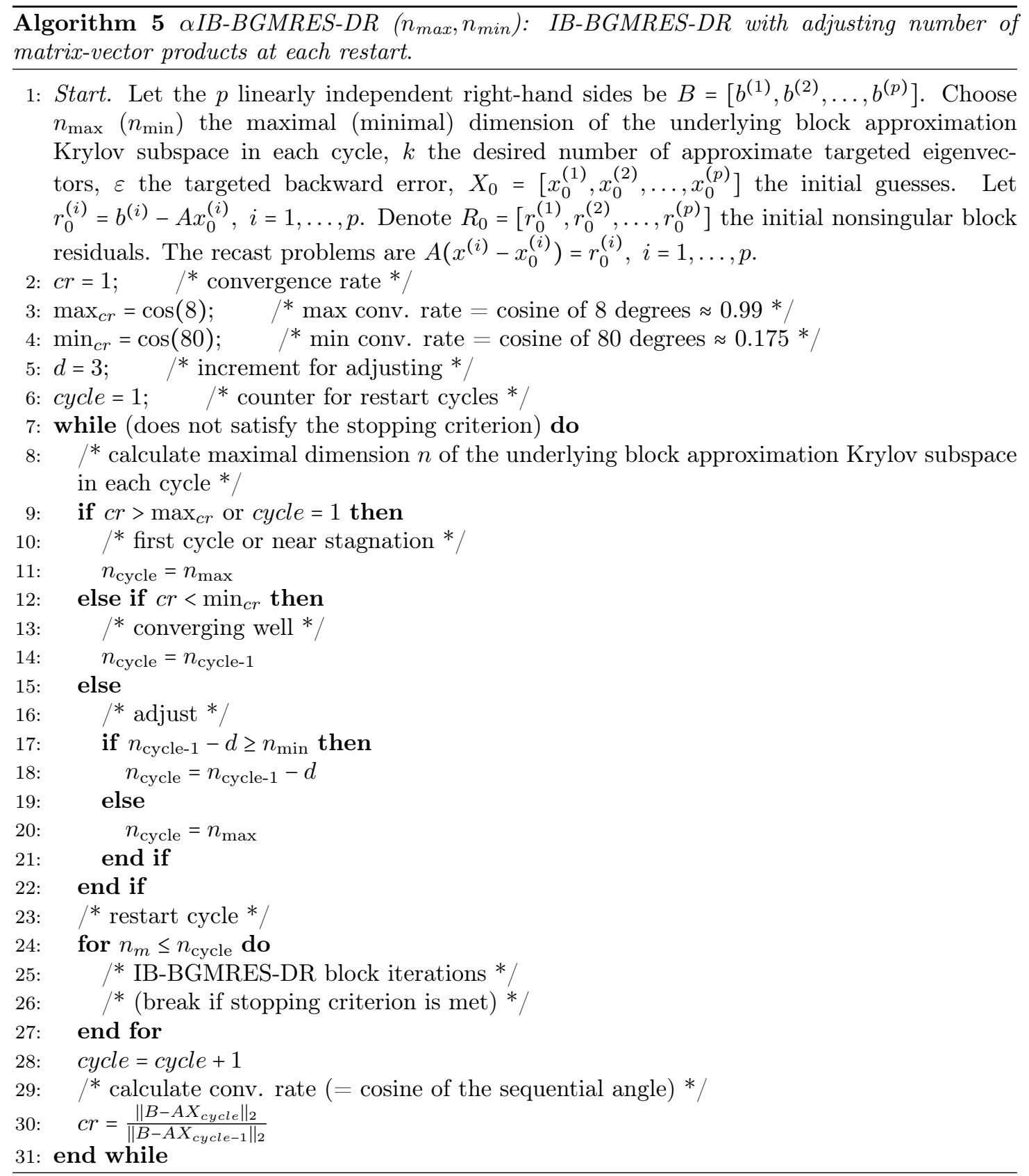




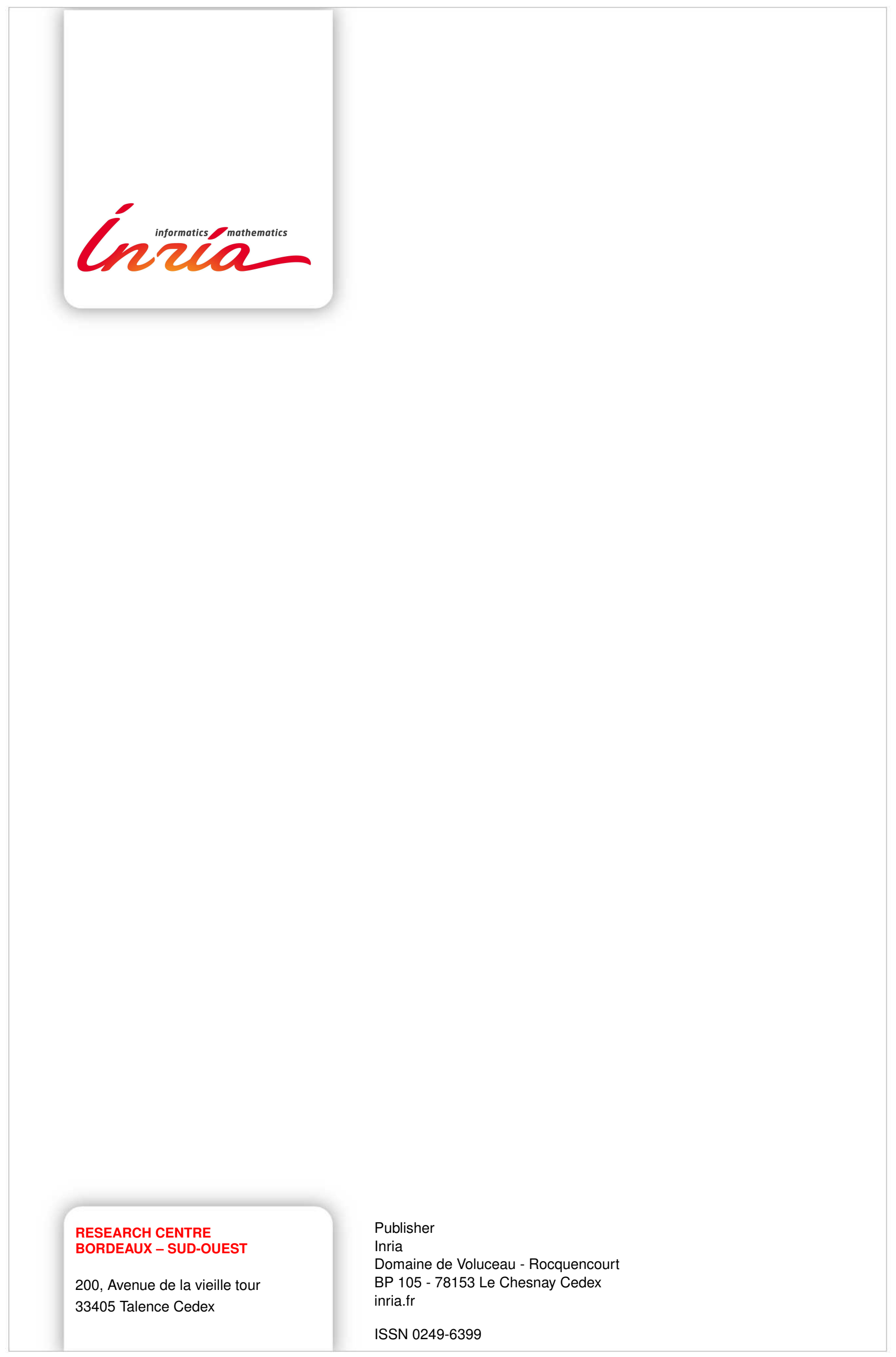

